\title{
Orbital effects on time delay interferometry for TianQin
}

\author{
Ming-Yue Zhou, ${ }^{1}$ Xin-Chun $\mathrm{Hu},{ }^{1}$ Bobing Ye, ${ }^{2}$ Shoucun $\mathrm{Hu},{ }^{3,4}$ \\ Dong-Dong Zhu, ${ }^{1}$ Xuefeng Zhang, ${ }^{2}$ Wei Su, ${ }^{1}$ and Yan Wang ${ }^{1, *}$ \\ ${ }^{1}$ MOE Key Laboratory of Fundamental Physical Quantities Measurements, \\ Hubei Key Laboratory of Gravitation and Quantum Physics, \\ PGMF, Department of Astronomy, and School of Physics, \\ Huazhong University of Science and Technology, Wuhan 430074, China \\ ${ }^{2}$ TianQin Research Center for Gravitational Physics \& School of Physics and Astronomy, \\ Sun Yat-sen University (Zhuhai Campus), Zhuhai 519082, China \\ ${ }^{3}$ CAS Key Laboratory of Planetary Sciences, Purple Mountain Observatory, \\ Chinese Academy of Sciences, Nanjing 210033, China \\ ${ }^{4}$ CAS Center for Excellence in Comparative Planetology, Hefei 230026, China
}

(Dated: May 3, 2021)

\begin{abstract}
The proposed space-borne laser interferometric gravitational wave (GW) observatory TianQin adopts a geocentric orbit for its nearly equilateral triangular constellation formed by three identical drag-free satellites. The geocentric distance of each satellite is $\approx 1.0 \times 10^{5} \mathrm{~km}$, which makes the armlengths of the interferometer be $\approx 1.73 \times 10^{5} \mathrm{~km}$. It is aimed to detect the GWs in $0.1 \mathrm{mHz}-1 \mathrm{~Hz}$. For space-borne detectors, the armlengths are unequal and change continuously which results in that the laser frequency noise is nearly $7-8$ orders of magnitude higher than the secondary noises (such as acceleration noise, optical path noise, etc.). The time delay interferometry (TDI) that synthesizes virtual interferometers from time-delayed one-way frequency measurements has been proposed to suppress the laser frequency noise to the level that is comparable or below the secondary noises. In this work, we evaluate the performance of various data combinations for both first- and secondgeneration TDI based on the five-year numerically optimized orbits of the TianQin's satellites which exhibit the actual rotating and flexing of the constellation. We find that the time differences of symmetric interference paths of the data combinations are $\sim 10^{-8} \mathrm{~s}$ for the first-generation TDI and $\sim 10^{-12} \mathrm{~s}$ for the second-generation TDI, respectively. While the second-generation TDI is guaranteed to be valid for TianQin, the first-generation TDI is possible to be competent for GW signal detection with improved stabilization of the laser frequency noise in the concerned GW frequencies.
\end{abstract}

* ywang12@hust.edu.cn 


\section{INTRODUCTION}

The detection of gravitational waves (GWs) from the coalescence of a stellar-mass black hole binary (GW150914) by advanced LIGO detectors [1] has opened up the era of observational GW astronomy. During the first two observing runs (O1 and $\mathrm{O} 2)[2]$ and the first half of O3 [3] of advanced LIGO and advanced Virgo, more than 50 compact binary coalescences, including the first observation of binary neutron star inspiral (GW170817) 4, have been detected. Both advanced LIGO and advanced Virgo will reach their design sensitivities in the coming years which can boost the detection of GW events to higher rate. In addition, the underground cryogenic GW telescope KAGRA [5] has recently joined in the advanced ground-based detector network.

The observational window of GW astronomy will be broadened to millihertz range $(0.1 \mathrm{mHz}-1 \mathrm{~Hz})$ by the proposed space-borne laser interferometers, such as Laser Interferometer Space Antenna (LISA) [6], TianQin 7], DECIGO [8], ASTROD-GW [9], gLISA [10, Taiji (ALIA descoped) [11] and BBO [12. Among these, LISA has been comprehensively studied and developed for more than three decades [13]. In 2017, it has been selected as ESA's L-3 mission Cosmic Vision programme with the theme of "the Gravitational Universe". LISA is scheduled for launch in early 2030s and will be operated concurrently with ESA's next generation Advanced Telescope for High ENergy Astrophysics (Athena). The latter will tremendously enhance the follow-up X-ray observation of the electromagnetic counterparts of LISA's GW source candidates [14. The successful flight of LISA Pathfinder has demonstrated the feasibility of the key technologies, such as gravity reference system and space laser interferometry, to be implemented in LISA [15, 16. The recent GRACE Follow-on mission has demonstrated the technologies to be used in the laser ranging interferometer (LRI) of LISA [17.

Similar to LISA, TianQin is comprised of three identical drag-free satellites that form a nearly equilateral triangular constellation [7. Each pair of satellites is linked by two one-way infrared laser beams which can be used, together with the intra-satellite laser links, to synthesize up to three Michelson interferometers. Distinct from LISA, TianQin adopts a geocentric orbit with an altitude of $10^{5} \mathrm{~km}$ from the geocenter, hence the armlength of each side of the triangle is approximately $1.73 \times 10^{5} \mathrm{~km}$. The detector plane formed by the three satellites faces to the galactic white dwarf binary RX J0806+1527 [18] (see Fig. 11). The guiding center of the constellation coincides with the geocenter, and the period of each satellite orbiting around the Earth is 3.65 days. Analytic approximation of the orbit coordinates for each satellite and the strain output of a Michelson interferometer for arbitrary incoming GWs are studied in [19]. A series of study on TianQin's orbit and constellation, including constellation stability optimization [20, orbital orientation and radius selection [21, eclipse avoidance [22, and the Earth-Moon's gravity disturbance evaluation [23], have been conducted. The recent progresses in the investigations of both science case and technological realization for TianQin can be found in [24 34]. A brief summary can be found in [35].

The GW sources in the $\mathrm{mHz}$ frequency regime are rich, which include coalescing supermassive black hole binaries (SMBHBs), ultracompact binaries in the Galaxy, extreme mass ratio inspirals (EMRIs), stochastic GW background, etc [36, 37]. For TianQin, preliminary studies on the detection rate of the SMBHBs [25], the associated parameter estimation accuracy based on the inspiral signals [24, and testing the no-hair theorem [26] and constraining the modified gravity [27] with the post-merger ringdown signals have been carried out. The prospects for detecting galactic double white dwarfs [30, EMRIs [29] and stellar-mass black hole binaries [28] with TianQin have been investigated.

Unlike the ground-based interferometers, the armlengths of a space-borne interferometer are unequal and varying in time. Therefore, the common mode laser frequency (or phase) noise, which is $7-8$ orders of magnitude higher than the secondary noises (such as optical path noise and acceleration noise), cannot be canceled out at the phasemeter where the delay lines from different paths are interfered. The time delay interferometry (TDI) has been proposed to suppress the laser frequency noise for space-borne interferometers to the level that is comparable or below the secondary noises, while conserving the GW content in the data stream, by synthesizing virtual interferometers with time-delayed oneway frequency measurements 38 46. The first-generation TDI is devised to remove the laser frequency noise in a static detector configuration. Multiple data combinations have been found which include the six-pulse combinations $(\alpha, \beta, \gamma, \zeta)$, the eight-pulse combinations, such as unequal-arm Michelson $(X, Y, Z)$, Relay $(U, V, W)$, Monitor $(E, F, G)$ and Beacon $(P, Q, R)$ (see examples in Fig. 44 [39, 47, and the optimal combinations $(A, E, T)$ [8].

All data combinations are aimed to make the lengths of the two symmetric interference paths in the synthesized interferometric measurements nearly equal. However, in the reality, this equality cannot be exactly satisfied due to the orbital dynamics of each satellite. The second-generation TDI has been proposed to further account for the rotation of the constellation and the linear variation of armlengths [44, 45, 49, which improves the length equality by judiciously splicing the first-generation interference paths [50. This results in more data combinations than the first-generation TDI.

The result from Synthetic LISA, based on the analytic approximation of LISA spacecraft's orbits [51, shows that the time differences of the symmetric interference paths are $10^{-6} \mathrm{~s}$ and $10^{-10} \mathrm{~s}$ for the first- and second-generation TDI, hence the latter must be adopted in LISA data analysis to comfortably cancel out the laser phase noises [52]. 
This is further confirmed by the detailed numerical simulations, based on the orbits optimized with CGC 2.7 ephemeris [53, of various first- and second-generation TDI data combinations for (e)LISA [54, 55]. Similar investigations have also been conducted for ASTROD-GW [56, 57] and Taiji [58].

In this work, we simulate the time differences of the symmetric interference paths of various TDI data combinations for TianQin. The optical paths are evaluated based on the numerical orbits of TianQin's satellites that have been optimized to meet the orbital stability requirements imposed by the long range space laser interferometry. Four types of the first-generation TDI data combinations show time differences of $\sim 10^{-8} \mathrm{~s}$ which makes them competent for GW signal detection for the frequencies $\lesssim 10^{-3} \mathrm{~Hz}$ and $\gtrsim 10^{-1} \mathrm{~Hz}$ given the stabilization of the laser frequency noise of $10 \mathrm{~Hz} / \sqrt{\mathrm{Hz}}$ in concerned frequency range. With an ample margin, the second-generation TDI data combinations with time differences of $\sim 10^{-12}$ s are warranted to reduce the laser frequency noise well below the secondary noises.

The rest of the paper is organized as follows. In section II] we discuss the orbit optimization for TianQin's satellites. The resulting numerical orbits interpolated by Chebyshev polynomials are subsequently used in the simulations of the time differences of the symmetric interference paths for various first- and second-generation TDI data combinations in section III A and III B, respectively. The paper is concluded in section IV

\section{CONSTELLATION OPTIMIZATION FOR TIANQIN}

The orbit of each satellite is primarily determined by the monopole gravitational field of the Earth. Besides, the perturbation from the multipole terms and relativistic post-Newtonian correction of the Earth gravitational field, the monople gravitational field of the Moon, the Sun, the major planets in the solar system, Pluto and large asteroids will also contribute. Dispersion from the Earth atmosphere and solar radiation are ignored due to the implementation of the drag-free control for the satellite platform. The minor eccentricity of the nominal Keplerian orbit along with time-dependent perturbation forces will induce variation of the armlengths, and flexing and breathing of the triangular constellation. On the other hand, the high precision space laser interferometry imposes requirements on the stability of the triangular constellation [7, 20]: (a) the armlength variation less than $0.5 \% \times\left(\sqrt{3} \times 10^{5}\right) \mathrm{km}$; $(\mathrm{b})$ the breathing angle (subtended by two arms) variation less than $0.1^{\circ}$ during the first two years and less than $0.2^{\circ}$ during the five years mission lifetime; (c) the relative range rate (Doppler velocity) less than $5 \mathrm{~m} / \mathrm{s}$ during the first two years and less than $10 \mathrm{~m} / \mathrm{s}$ in five years.

\section{A. Calculation of the satellite orbits}

In this work, the geocentric ecliptic coordinate system $(x, y, z)$ shown in Fig. 1 has been adopted in the calculation and optimization of the orbits for TianQin's satellites. This choice is different from [19] in which, for the sake of calculating the antenna response of TianQin to GWs, the heliocentric ecliptic coordinate system is used. The gravitational field of the Earth is described in the Earth-fixed reference coordinate system WGS84 [59].

From the six initial Keplerian elements $\sigma_{0}=\left(a_{k}, e_{k}, i_{k}, \Omega, \omega, M_{k}\right)$ of the $k$-th $(k \in\{1,2,3\})$ satellite, we can obtain the initial Cartesian coordinates in the geocentric ecliptic coordinate system as follows [60]:

$$
\begin{aligned}
& x_{k}=l_{1} a_{k}\left(\cos E_{k}-e_{k}\right)+l_{2} a_{k} \sqrt{1-e_{k}^{2}} \sin E_{k}, \\
& y_{k}=m_{1} a_{k}\left(\cos E_{k}-e_{k}\right)+m_{2} a_{k} \sqrt{1-e_{k}^{2}} \sin E_{k}, \\
& z_{k}=n_{1} a_{k}\left(\cos E_{k}-e_{k}\right)+n_{2} a_{k} \sqrt{1-e_{k}^{2}} \sin E_{k} .
\end{aligned}
$$

Here $a_{k} \approx 1.0 \times 10^{5} \mathrm{~km}$ is the semi-major axis, and $e_{k} \approx 0$ is the orbit eccentricity. The eccentric anomaly $E_{k}$ can be obtained by the Newton's iteration method [61:

$$
E_{n+1, k}=E_{n, k}+\frac{M_{k}-E_{n, k}+e_{k} \sin E_{n, k}}{1-e_{k} \cos E_{n, k}}, \quad(n=0,1,2, \cdots)
$$

where $n$ is the index of the iteration and $E_{0, k}=M_{k}$. We set the mean anomaly $M_{k}=\varpi_{k}\left(t-t_{0}\right)+120^{\circ} \times(k-1)+60^{\circ}$ in order to arrange the three satellites into a nearly equilateral triangle constellation. $\varpi_{k}=\sqrt{G M_{\oplus} / a_{k}^{3}}$ represents the angular velocity of the satellite, where $M_{\oplus}$ is the Earth mass. The surrogate variables $\left(l_{1,2}, m_{1,2}, n_{1,2}\right)$ in Eq. 1 
are defined as follows:

$$
\begin{aligned}
& l_{1}=\cos \Omega \cos \omega-\sin \Omega \sin \omega \cos i_{k}, \\
& m_{1}=\sin \Omega \cos \omega+\cos \Omega \sin \omega \cos i_{k}, \\
& n_{1}=\sin \omega \sin i_{k} ; \\
& l_{2}=-\cos \Omega \sin \omega-\sin \Omega \cos \omega \cos i_{k}, \\
& m_{2}=-\sin \Omega \sin \omega+\cos \Omega \cos \omega \cos i_{k}, \\
& n_{2}=\cos \omega \sin i_{k} .
\end{aligned}
$$

Here we set the argument of periapsis (the angle between ascending node and perigee) $\omega=0$. The three satellites form a detector plane (subtended by $x^{\prime}$ and $y^{\prime}$ axes in Fig. 1), the normal of which $\left(z^{\prime}\right.$ axis) points toward the reference source RX J0806+1527. It thus indicates that the inclination of the detector plane $i_{k}=94.7^{\circ}$ and the longitude of the ascending node (the angle between $x$ axis and $x^{\prime}$ axis) $\Omega=210.5^{\circ}$.

Finally, through coordinate transformation, we obtain the expression for the initial positions of the satellites in geocentric equatorial coordinates. Here we choose obliquity of the ecliptic $\varepsilon=23^{\circ} 26^{\prime \prime}$.

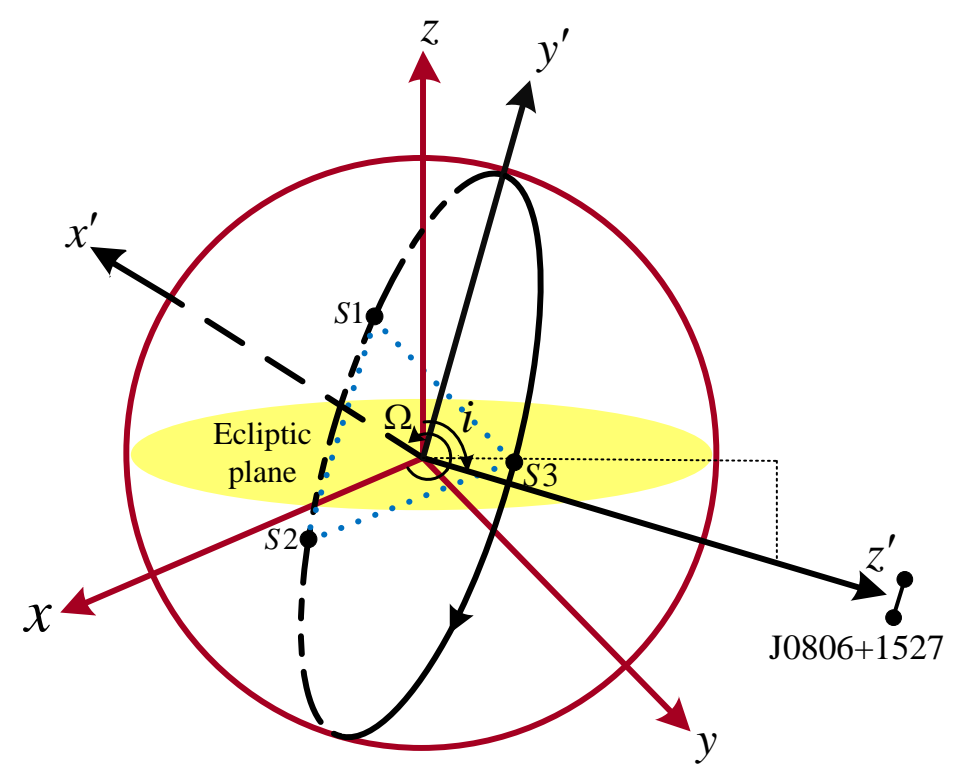

FIG. 1. $(x, y, z)$ represents the geocentric ecliptic coordinate system, $x$ axis points toward the mean equinox of J2000. $\left(x^{\prime}, y^{\prime}, z^{\prime}\right)$ represents the orbit coordinate system of the detector, $x^{\prime}$ axis points toward the ascending node of the satellites and $z^{\prime}$ axis points toward the reference source RX J0806+1527. $\Omega$ is the angle between $x$ and $x^{\prime}, i$ is the inclination of the detector plane.

The subsequent evolution of the satellites' orbits, after setting up the initial condition, is determined by the dynamic equation [59] (for clarity we ignore the satellite index $k$ hereafter),

$$
\ddot{\mathbf{r}}=\ddot{\mathbf{r}}_{\mathrm{NB}}+\ddot{\mathbf{r}}_{\mathrm{NS}}+\ddot{\mathbf{r}}_{\mathrm{PN}},
$$

where $\mathbf{r}$ represents the position vector of the satellite relative to the geocenter. The Newtonian body term is

$$
\ddot{\mathbf{r}}_{\mathrm{NB}}=-G M_{\oplus} \mathbf{r} / r^{3}-\sum_{i=1}^{10} G m_{i}\left(\boldsymbol{\Delta}_{i} / \Delta_{i}^{3}+\mathbf{r}_{i} / r_{i}^{3}\right),(i=1,2,3, \cdots, 10) .
$$

Here $G$ is the gravitational constant. $m_{i}$, in the ascending order of $i$, denotes the masses of the seven major planets, Pluto, the Sun and the Moon. $\mathbf{r}_{i}$ and $\boldsymbol{\Delta}_{i}=\mathbf{r}-\mathbf{r}_{i}$ are the position vectors of the $i$-th perturbing body relative to the geocenter and the satellite, respectively. $r_{i}=\left|\mathbf{r}_{i}\right|$ and $\Delta_{i}=\left|\boldsymbol{\Delta}_{i}\right|$. The positions of the planets, the Sun, the Moon are extracted from the ephemeris DE421 62]. $\ddot{\mathbf{r}}_{\mathrm{NS}}$ includes the sectorial and tesseral harmonic terms of the Earth's non-spherical gravitational perturbation, respectively. The first-order post-Newtonian correction term [63 65] is

$$
\ddot{\mathbf{r}}_{\mathrm{PN}}=\frac{G M_{\oplus}}{c^{2} r^{2}}\left[\left(4 G M_{\oplus} / r-v^{2}\right)(\mathbf{r} / r)+4(\mathbf{r} \cdot \dot{\mathbf{r}})(\dot{\mathbf{r}} / r)\right],
$$


TABLE I. The values of the elements of the optimized orbit $\bar{\sigma}_{0}^{*}$ for the three satellites at the initial epoch $t_{0}=$ MJD 64104.5 .

\begin{tabular}{cccc}
\hline \hline & $\begin{array}{c}\text { Semi-major axis } \\
a(\mathrm{~km})\end{array}$ & $\begin{array}{c}\text { Inclination } \\
i\left({ }^{\circ}\right)\end{array}$ & $\begin{array}{c}\text { Eccentricity } \\
e\end{array}$ \\
\hline S1 & 99995.0717 & 94.7473 & $2.8483 \times 10^{-4}$ \\
\hline S2 & 100010.8914 & 95.7094 & 0 \\
\hline S3 & 99992.5623 & 94.6469 & $2.0876 \times 10^{-4}$ \\
\hline
\end{tabular}

where $v=|\dot{\mathbf{r}}|$, and $c$ is the speed of light.

Once set the orbit elements at an initial epoch, for example $t_{0}=$ MJD 64104.5 (May 22 2034 12:00:00 TDB), we can numerically integrate Eq. 4 by Runge-Kutta 7(8) method to obtain $\mathbf{r}$ and $\dot{\mathbf{r}}$ at subsequent time stamps $t_{i}$ $(i=1,2, \cdots, N)$, where $N=T / \Delta t$ and $T=5 \mathrm{yr}$. The integration time stepsize $\Delta t=1 \mathrm{hr}$, which is chosen such that the total computational error (combining the accumulated round-off error from arithmetic operations of double-precision float number and the truncation error of Runge-Kutta algorithm) approaches its minimum.

\section{B. The constellation optimization}

In order to fulfill the aforementioned requirements on orbit stability, we try to find a set of initial orbit elements in the vicinity of the fiducial ones such that during the mission lifetime the armlength variation measured by the sum of squared length differences of the three arms reaches a global minimum in the parameter space. It has been proved effective in practice to only search in the nine dimensional parameter space spanned by $\bar{\sigma}_{0}=\left(a_{k}, i_{k}, e_{k}\right), k \in\{1,2,3\}$ [66]. Thus, the constellation optimization problem can be formulated as follows,

$$
\bar{\sigma}_{0}^{*}=\underset{\bar{\sigma}_{0} \in \mathbb{D}}{\arg \min } \mathcal{O}\left(\bar{\sigma}_{0}\right)=\underset{\bar{\sigma}_{0} \in \mathbb{D}}{\arg \min } \sum_{k=1}^{3} \sum_{i=1}^{N}\left(L_{k, i}-L_{k, 0}\right)^{2},
$$

where $L_{k, i}$ represents the length of the arm opposite to the $k$-th satellite at time $t_{i}$, and $L_{k, 0}$ is the armlength at the initial epoch. $\mathbb{D}$ is the search space of the optimization with the ranges: $a_{k} \in[0.999,1.001] \times 10^{5} \mathrm{~km}, e_{k} \in\left[0,1 \times 10^{-3}\right]$, $i_{k} \in\left[93.2^{\circ}, 96.2^{\circ}\right]$.

The objective function $\mathcal{O}\left(\bar{\sigma}_{0}\right)$ may have a highly multi-modal landscape owning a forest of local minima. Therefore any deterministic local optimizer would locate a suboptimal solution. On the other hand, a direct grid search will be computationally prohibitive due to the high dimension $(\operatorname{dim}(\mathbb{D})=9)$. Instead, the optimization methods including some randomness can be used to effectively pinpoint the global minimizer in $\mathcal{O}\left(\bar{\sigma}_{0}\right)$. In this work, we use one of the stochastic optimization methods based on emulating biological groups, namely the particle swarm optimization (PSO) 67. PSO has been applied in many fields [68, including gravitational wave data analysis for detecting and estimating compact binary coalescence signals in a network of ground-based laser interferometers [69, 70] and continuous waves in pulsar timing arrays [71, 72].

The resulting $\bar{\sigma}_{0}^{*}$ for each satellite is listed in Table 1 . The variations of the armlengths, the variations of breathing angles, Doppler velocities and pointing deviation (from the direction of RX J0806+1527) of the satellite constellation during 5-yr mission lifetime are shown in Fig. 2. We can see that the optimized orbits satisfy the aforementioned requirements on the constellation stability with some margins. Note that, although the constellation optimization method is different from the three-step scheme adopted in [20, the results, in terms of the initial elements and performance of constellation optimization is virtually consistent (see Fig. 1 in [20]).

\section{Interpolation of the orbit}

The orbit coordinates of the satellites can be represented as finite sequences with sampling interval of $\Delta t$. As it will become more clear in Sec. III , the orbit positions and velocities at arbitrary time points within $\left(t_{i}, t_{i+1}\right)$ are needed in generating the TDI data combinations. In this case, we use Chebyshev polynomials to approximate the orbit of each satellite at $t \in\left(t_{i}, t_{i+1}\right)$. This method is stable and has been widely used in high precision ephemeris interpolation, such as the DE series ephemerides developed by Jet Propulsion Laboratory (JPL) 73. Here, Chebyshev polynomials up to 15 orders are adopted and the sampling interval of the fitted data is 0.5 day with interpolation precision of $\sim 10^{-10} \mathrm{~km}$. The weight of position and velocity is $1: 0.4$, which turns out to be the optimal choice to calculate the positions of the planets in the solar system [73. The function approximation algorithm finds the best-fit coefficients of Chebyshev polynomials by minimizing the variance of the residuals between the data and the model 74 . 


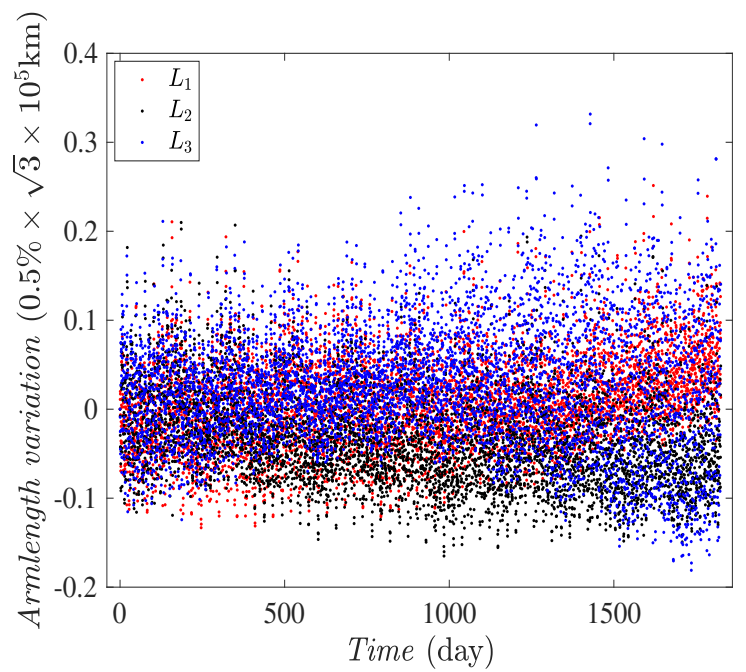

(a)

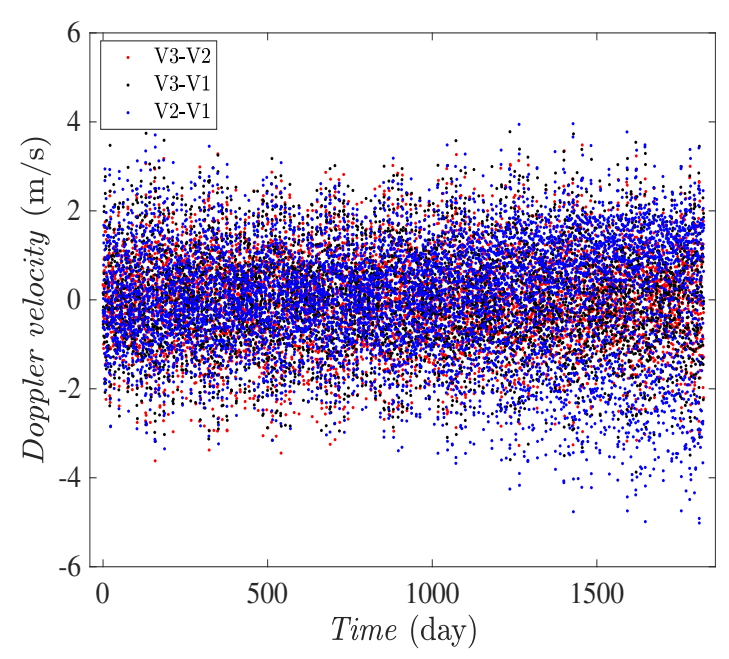

(c)

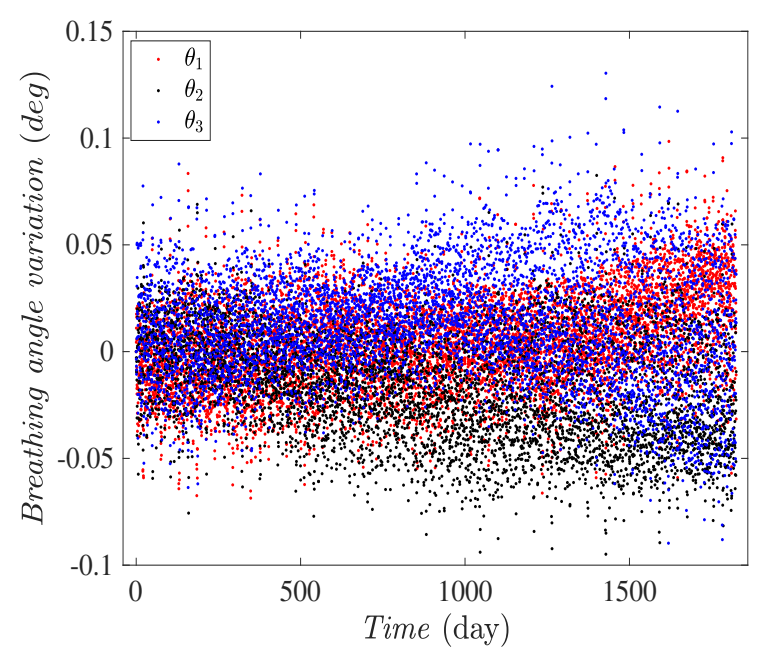

(b)

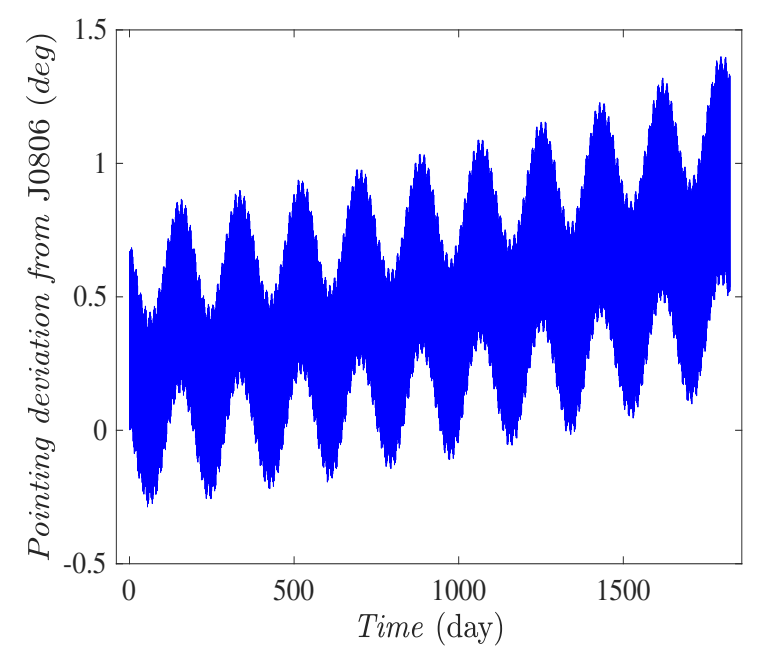

(d)

FIG. 2. (a) The variations of armlengths; $(b)$ The variations of breathing angles; $(c)$ Doppler velocities; $(d)$ Pointing deviation from J0806+1527 during 5-yr mission lifetime.

\section{SIMULATION OF TIME DIFFERENCES FOR TDI DATA COMBINATIONS}

In this work, we assume that the lasers on the two optical benches housed in a satellite are locked in phase, so we only consider the inter-satellite fractional frequency measurements ' $y$ ' which account for the cancellation of the laser frequency noise. Following [47, the naming convention of the interference arms is shown in Fig. 3 .

\section{A. Time differences for the first-generation TDI}

The first-generation TDI has 15 interference data combinations, namely $(\alpha, \beta, \gamma),(X, Y, Z),(U, V, W),(E, F, G)$, and $(P, Q, R)$. Five representative combinations are shown in Fig. 4 . The other subtypes are different in the starting satellite of light paths and their combinations can be obtained by cyclic permutation of the indices for satellites: $1 \rightarrow 2 \rightarrow 3 \rightarrow 1$. As an example, the Doppler data for the Sagnac-type $\alpha$ combination is as follows:

$$
\alpha=y_{23,13}+y_{12,3}+y_{31}-y_{21}-y_{13,2^{\prime}}-y_{32,1^{\prime} 2^{\prime}},
$$




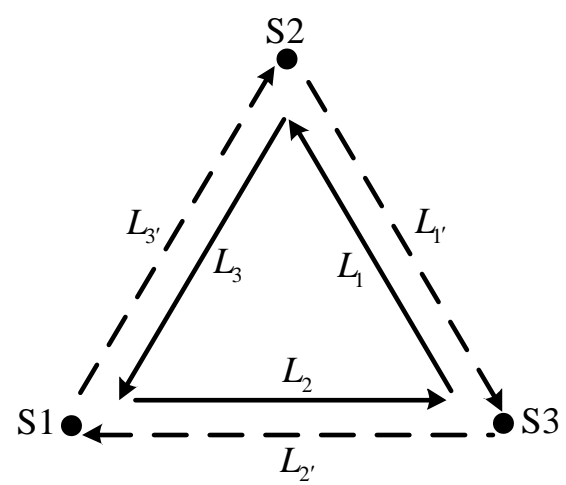

FIG. 3. The labels of the satellites and the interference arms.

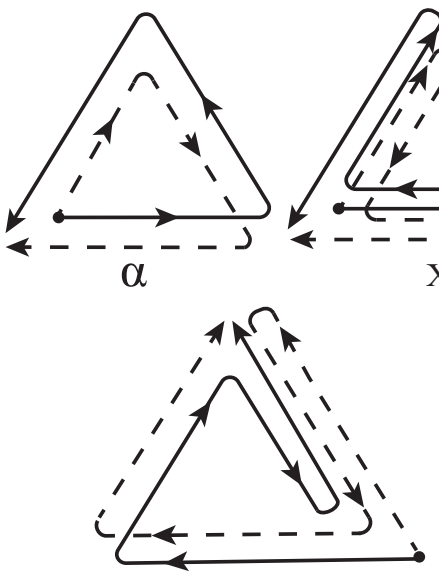

$\mathrm{U}$
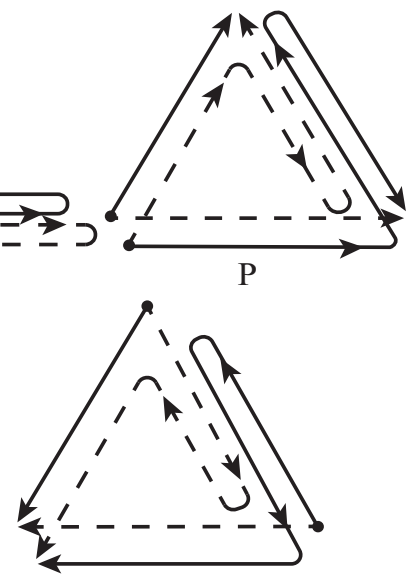

$\mathrm{E}$

FIG. 4. Schematic diagram of the first-generation TDI combinations.

where ',' marks the time delay of the laser beam traveling along an arm. $y_{23,13}=y_{23}\left(t-L_{1}-L_{3}\right)$, which represents the time-delayed fractional frequency fluctuation time series measured at reception by S3 with transmission from S1 along arm 2. Here and hereafter, we set $c=1$. As in Fig. 4, the clockwise (1-2-3-1) and the counter clockwise (1-3-2-1) light paths interfere at S1 at time $t$. While, their initial times of emission at S1 are $t-L_{3^{\prime}}-L_{1^{\prime}}-L_{2^{\prime}}$ and $t-L_{2}-L_{1}-L_{3}$, respectively. Inserting the fractional frequency fluctuation $C_{i}(t)$ of the laser on $\mathrm{S} i$ into Eq. 8 , we obtain:

$$
\begin{aligned}
\delta C_{\alpha}(t)= & {\left[C_{1}\left(t-L_{2}-L_{1}-L_{3}\right)-C_{3}\left(t-L_{1}-L_{3}\right)\right]+\left[C_{3}\left(t-L_{1}-L_{3}\right)-C_{2}\left(t-L_{3}\right)\right] } \\
& \left.+\left[C_{2}\left(t-L_{3}\right)-C_{1}(t)\right)\right]-\left[C_{3}\left(t-L_{2^{\prime}}\right)-C_{1}(t)\right] \\
& -\left[C_{2}\left(t-L_{1^{\prime}}-L_{2^{\prime}}\right)-C_{3}\left(t-L_{2^{\prime}}\right)\right] \\
& -\left[C_{1}\left(t-L_{3^{\prime}}-L_{1^{\prime}}-L_{2^{\prime}}\right)-C_{2}\left(t-L_{1^{\prime}}-L_{2^{\prime}}\right)\right] \\
& =C_{1}\left(t-L_{2}-L_{1}-L_{3}\right)-C_{1}\left(t-L_{3^{\prime}}-L_{1^{\prime}}-L_{2^{\prime}}\right) .
\end{aligned}
$$

The first-generation TDI assumes a fixed detector constellation in space and the armlengths are unequal but constant, thus Eq. 9 can be canceled out exactly. However, in reality, the armlengths are time varying due to the actual rotating and flexing of the constellation. To the first order of $L_{l}(t)$, Eq. 9 can be expanded as:

$$
\begin{aligned}
\delta C_{\alpha}(t) & \simeq \dot{C}_{1}(t)\left[-L_{2}+\dot{L}_{2}\left(L_{1}+L_{3}\right)-L_{1}+\dot{L}_{1} L_{3}-L_{3}\right. \\
& \left.+L_{3^{\prime}}-\dot{L}_{3^{\prime}}\left(L_{1^{\prime}}+L_{2^{\prime}}\right)+L_{1^{\prime}}-\dot{L}_{1^{\prime}}\left(L_{2^{\prime}}\right)+L_{2^{\prime}}\right]
\end{aligned}
$$


where both $L_{l}$ and $\dot{L}_{l}$ are evaluated at time $t$. When only the zero order of $L_{l}(t)$ is concerned, as in the first-generation TDI, Eq. 10 vanishes. The data combinations that have this nature are called L-closed [50]. Therefore, the level of laser frequency noise cancellation is determined by how well we can synthesize equal-length virtual paths, such as the two paths for the $\alpha$ combination, in the construction of virtual interferometers. In other words, from Eq. 9, we can see that the magnitude of the residual laser noise can be measured by the time difference of the two interference paths, which, for the $\alpha$ combination, is

$$
\Delta t_{\alpha}=L_{2,13}+L_{1,3}+L_{3}-L_{2^{\prime}}-L_{1^{\prime}, 2^{\prime}}-L_{3^{\prime}, 1^{\prime} 2^{\prime}}
$$

Here, $L_{2,13}=L_{2}\left(t-L_{1}(t)-L_{3}(t)\right)$ is the length of $L_{2}$ at time $t-L_{1}(t)-L_{3}(t)$. Through the armlengths obtained in Sec. II B, we can calculate the time differences of interference paths, which can be used to analyze the level of the laser frequency noise cancellation. Fig. 5 presents the result for the $\alpha$ combination in the five years mission lifetime. We can see the net time difference of the two opposite paths induced by the Sagnac effect $\Delta t_{\text {Sag }}=4 \vec{\Omega} \cdot \vec{A}[49$, 75 . With the area $|\vec{A}|=\sqrt{3} L^{2} / 4\left(L \simeq \sqrt{3} \times 10^{5} \mathrm{~km}\right)$ and the angular velocity of the constellation $|\vec{\Omega}|=1 / 3.65 \mathrm{cycle} /$ day for TianQin, $\Delta t_{\text {Sag }} \simeq 1.15 \times 10^{-5}$ s. Therefore, the result of this data combination is about three orders of magnitude worse than the other first-generation data combinations below.

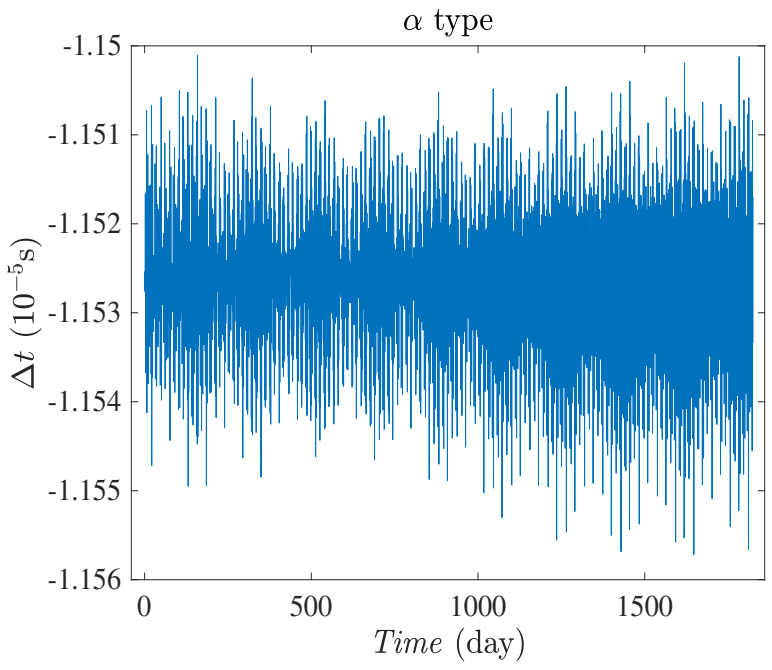

FIG. 5. Simulation of the time difference for the first-generation TDI $\alpha$ combination.

Following the same procedure, the time differences for the other types can be written as:

$$
\begin{aligned}
& \Delta t_{X}=L_{3^{\prime}, 322^{\prime}}+L_{3,22^{\prime}}+L_{2,2^{\prime}}+L_{2^{\prime}}-L_{3}-L_{3^{\prime}, 3}-L_{2^{\prime}, 3^{\prime} 3}-L_{2,2^{\prime} 3^{\prime} 3}, \\
& \Delta t_{U}=L_{2^{\prime}, 3^{\prime} 1^{\prime} 1}+L_{3^{\prime}, 1^{\prime} 1}+L_{1^{\prime}, 1}+L_{1}-L_{3^{\prime}}-L_{2^{\prime}, 3^{\prime}}-L_{1^{\prime}, 2^{\prime} 3^{\prime}}-L_{1,1^{\prime} 2^{\prime} 3^{\prime}}, \\
& \Delta t_{P}=L_{3^{\prime}, 1^{\prime} 12}+L_{1^{\prime}, 12}+L_{1,2}-L_{3^{\prime}, 2}+L_{2,3^{\prime}}-L_{1^{\prime}, 3^{\prime}}-L_{1,1^{\prime} 3^{\prime}}-L_{2,11^{\prime} 3^{\prime}}, \\
& \Delta t_{E}=L_{1^{\prime}, 13}+L_{1,3}+L_{3}-L_{2^{\prime}}-L_{1^{\prime}, 2^{\prime}}-L_{1,1^{\prime} 2^{\prime}}+L_{2^{\prime}, 1^{\prime} 1}-L_{3,11^{\prime}} .
\end{aligned}
$$

The corresponding results are shown in Fig. 6. The time differences for the first-generation TDI $X, U, P$ and $E$ data combinations are $\sim 10^{-8} \mathrm{~s}$.

The TDI data combinations can be considered effective when, taking the $\alpha$ combination as an example, $\left|\delta \tilde{C}_{\alpha}(f)\right|^{2} \leq$ $S_{\alpha}(f)$. Here,

$$
\left|\delta \tilde{C}_{\alpha}(f)\right|^{2}=4 \pi^{2} f^{2}\left|\tilde{C}_{1}\right|^{2} \delta t_{\alpha}^{2},
$$

which can be obtained by using the derivative theorem of Fourier transform for Eq. $9\left[52 . \delta t_{\alpha} \equiv \sup \left\{\left|\Delta t_{\alpha}(t)\right|\right\}\right.$. $S_{\alpha}(f)=\left[4 \sin ^{2}(3 \pi f L)+24 \sin ^{2}(\pi f L)\right] S_{y}^{\text {accel }}+6 S_{y}^{\text {opt }}$ is the secondary noise power spectral density (PSD) for the $\alpha$ combination [39], where $S_{y}^{\text {accel }}=2.8 \times 10^{-49}(f / 1 \mathrm{~Hz})^{-2} \mathrm{~Hz}^{-1}$ and $S_{y}^{\text {opt }}=4.4 \times 10^{-40}(f / 1 \mathrm{~Hz})^{2} \mathrm{~Hz}^{-1}$ are the PSDs of the acceleration noise and the optical path noise [39] assuming the one-sided amplitude spectra of the acceleration noise and the optical path noise of a single link are $1 \times 10^{-15} \mathrm{~m} \mathrm{~s}^{-2} / \sqrt{\mathrm{Hz}}$ and $1 \mathrm{pm} / \sqrt{\mathrm{Hz}}$, respectively, for TianQin [7, 19. $\left|\tilde{C}_{1}\right|^{2}=1.26 \times 10^{-27} \mathrm{~Hz}^{-1}$ is the fractional laser frequency fluctuation (corresponding to the raw laser frequency noise of $10 \mathrm{~Hz} / \sqrt{\mathrm{Hz}})$ [7, 39], which is assumed to be white in the concerned frequency range. Then, we find that the required time difference $\delta t_{\alpha} \leq 2.3 \times 10^{-7} \mathrm{~s}$. 


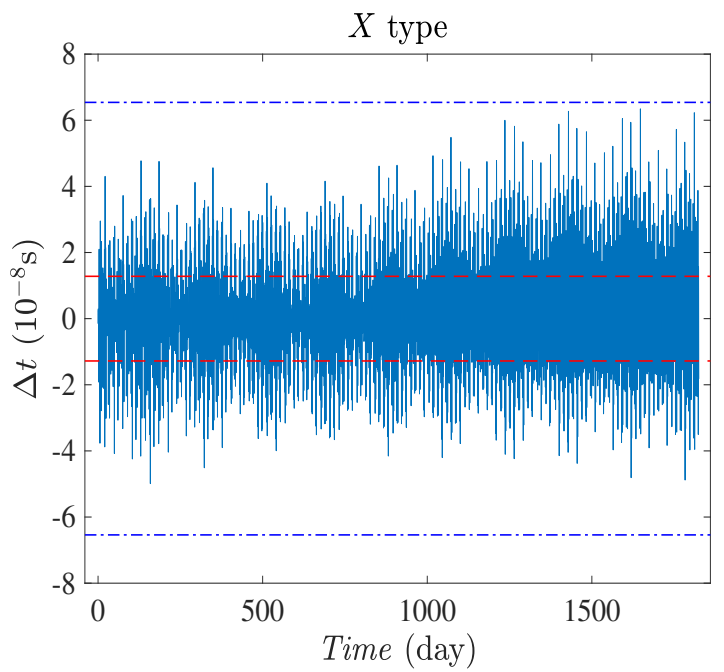

(a)

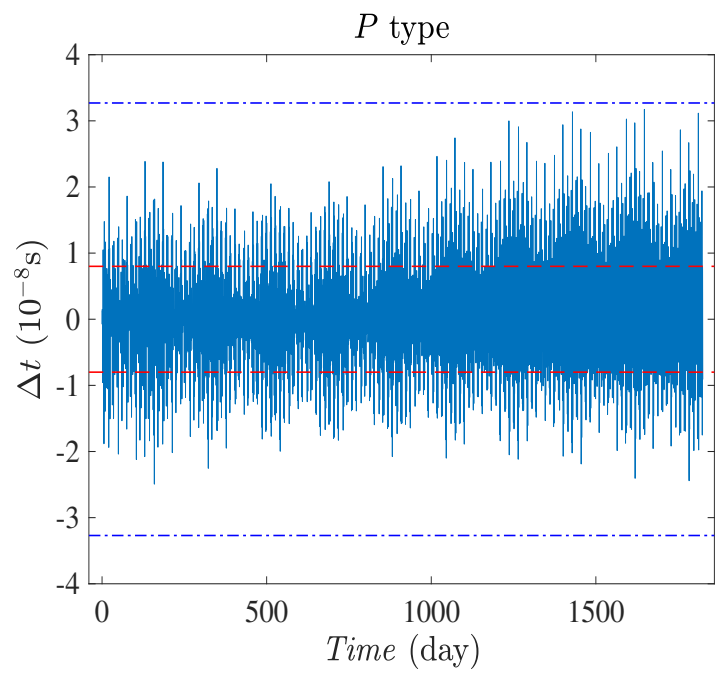

(c)

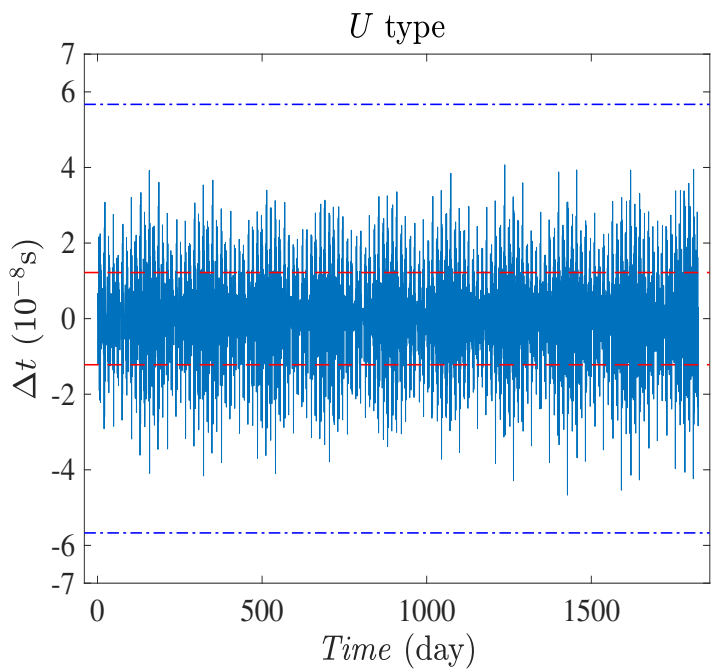

(b)

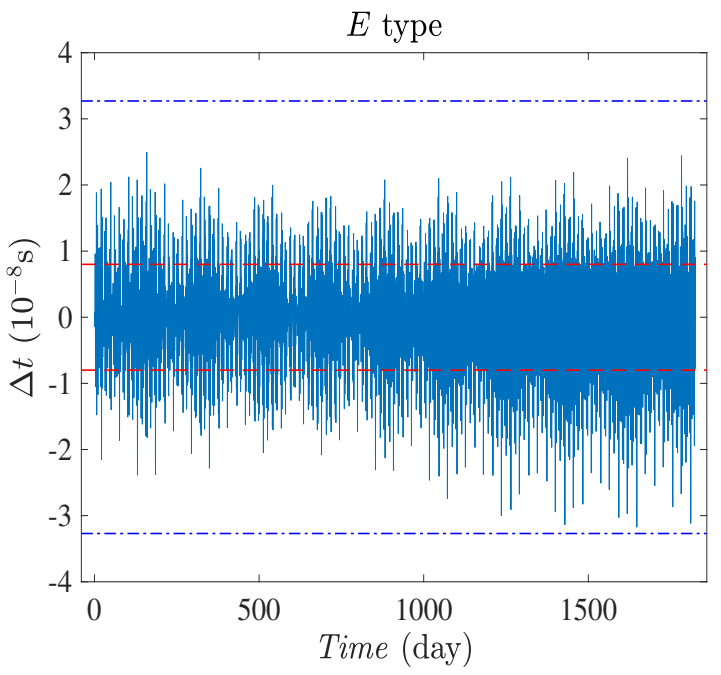

(d)

FIG. 6. Simulation of the time differences for the first-generation TDI $X, U, P$ and $E$ combinations (blue solid line). In each panel, the red dash lines represent the lowest required time differences for each combinations at $f \approx 10^{-2.2} \mathrm{~Hz}(\mathrm{c} . f$. right panel of Fig. 7). For comparison, the blue dash-dot lines represent the ones at $f=10^{-3} \mathrm{~Hz}$.

Similarly, for the $X, U, P$, and $E$ combinations with the corresponding secondary noise PSDs as follows,

$$
\begin{aligned}
S_{X}(f) & =\left[4 \sin ^{2}(4 \pi f L)+32 \sin ^{2}(2 \pi f L)\right] S_{y}^{\text {accel }}+16 \sin ^{2}(2 \pi f L) S_{y}^{\mathrm{opt}}, \\
S_{U}(f) & =\left[8 \sin ^{2}(3 \pi f L)+12 \sin ^{2}(2 \pi f L)+24 \sin ^{2}(\pi f L)\right] S_{y}^{\text {accel }} \\
& +\left[4 \sin ^{2}(3 \pi f L)+8 \sin ^{2}(2 \pi f L)+4 \sin ^{2}(\pi f L)\right] S_{y}^{\text {opt }}, \\
S_{P}(f) & =\left[4 \sin ^{2}(2 \pi f L)+32 \sin ^{2}(\pi f L)\right] S_{y}^{\text {accel }}+\left[8 \sin ^{2}(2 \pi f L)+8 \sin ^{2}(\pi f L)\right] S_{y}^{\text {opt }}, \\
S_{E}(f) & =S_{P}(f) .
\end{aligned}
$$

The required time differences are shown in the right panel of Fig. 7

The PSDs of the secondary noises and the residual laser frequency noises for the $X, U, P$, and $E$ combinations are shown in the left panel of Fig. 7. For the latter, the actual time differences $\delta t$ from Fig. 6 6 are used. We can see that the first-generation TDI data combinations cannot suppress the laser frequency noises below the secondary noises for $10^{-3} \lesssim f \lesssim 10^{-1} \mathrm{~Hz}$; while they are effective at the frequencies $f \lesssim 10^{-3} \mathrm{~Hz}$ and $f \gtrsim 10^{-1} \mathrm{~Hz}$. 


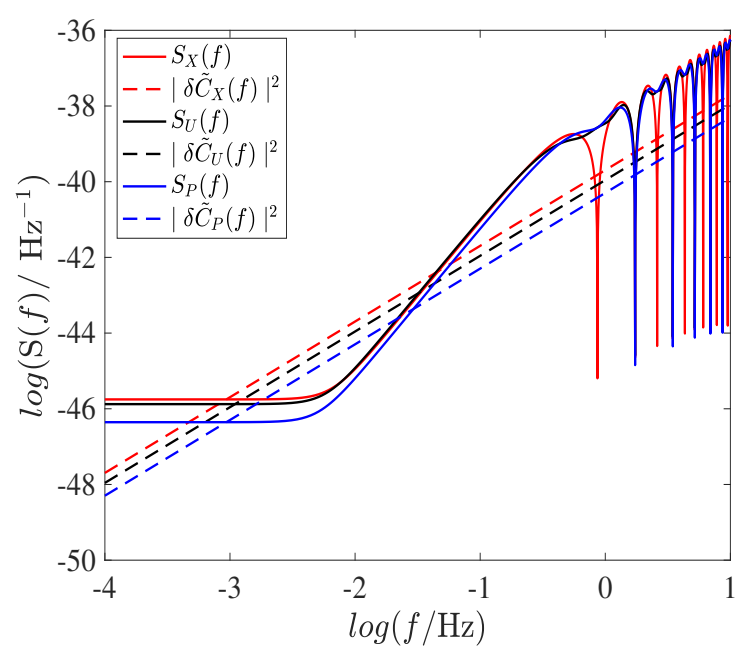

(a)

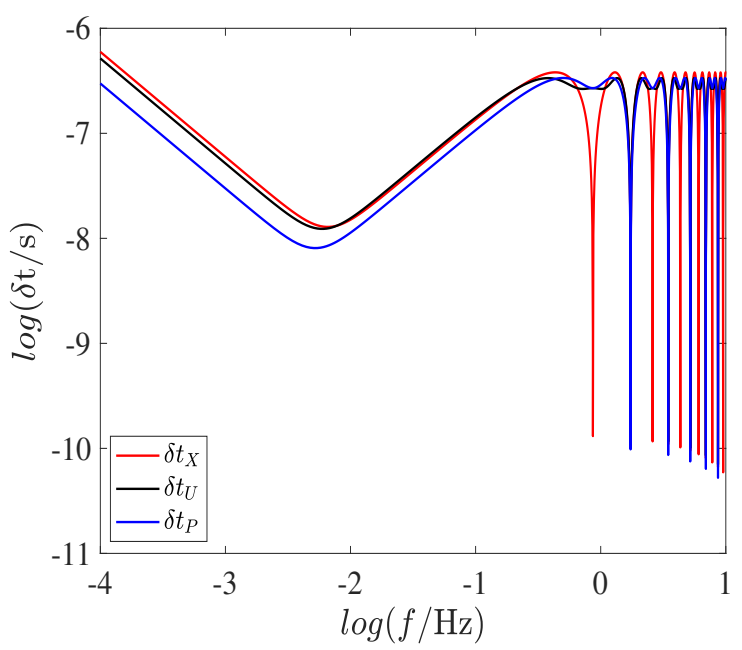

(b)

FIG. 7. Left panel shows the secondary noise (solid line) and the residual laser frequency noise (dotted line) for the firstgeneration $X, U, P$ and $E$ combinations. The lines for $P$ and $E$ are overlapped, only the ones for $P$ are shown. Right panel shows the required time differences for the corresponding combinations.

\section{B. Time differences for the second-generation TDI}

The second-generation TDI can suppress the laser frequency noise further by canceling the $L_{l}$ and $L_{l} \dot{L}_{m}$ terms in Eq. 10. The data combinations that have this nature are called $\dot{L}$-closed, for which the interference paths can be found through splicing the first-generation interference paths [44. Taking the $\alpha$ combination as an example, a new path 1 (1-3-2-1-2-3-1) can be synthesized by splicing the path 1 (1-3-2-1) and path 2 (1-2-3-1) of the first-generation $\alpha$; a new path 2 (1-2-3-1-3-2-1) is in a reversed order of the satellites. Specifically, this process can be expressed as:

$$
\begin{aligned}
& \overrightarrow{{ }_{1} 1_{2} 3_{1}{ }_{1}} \overleftarrow{2_{3}^{\prime} 1_{2}^{\prime} 3_{1}^{\prime}}+\overrightarrow{{ }_{1} 3_{2}^{\prime} 1_{3}^{\prime} 2_{1}^{\prime}} \overleftrightarrow{3_{2} 1_{3} 2_{1}} \Longrightarrow \overrightarrow{213\left[3^{\prime} 1^{\prime} 2^{\prime}\right.} \overleftrightarrow{312] 2^{\prime} 1^{\prime} 3^{\prime}} \quad(\alpha 12-1) \\
= & y_{23 ; 133^{\prime} 1^{\prime} 2^{\prime}}+y_{12 ; 33^{\prime} 1^{\prime} 2^{\prime}}+y_{31 ; 3^{\prime} 1^{\prime} 2^{\prime}}+y_{32 ; 1^{\prime} 2^{\prime}}+y_{13 ; 2^{\prime}}+y_{21} \\
& -y_{31}-y_{12 ; 3}-y_{23 ; 13}-y_{21 ; 213}-y_{13 ; 2^{\prime} 213}-y_{32 ; 1^{\prime} 2^{\prime} 213} .
\end{aligned}
$$

Here, $y_{23 ; 13}=y_{23}\left(t-L_{1}\left(t-L_{3}(t)\right)-L_{3}(t)\right)$. Note that we use ';' here to represent the time delay in the second generation. Two laser beams passing along ' $\longrightarrow$ ' and ' $\longleftarrow$ ' interfere at time $t$. The numbers below each arrow represent the interference arms, the subscripts on the left hand side represent the indices of satellites. On the right hand side, $\overrightarrow{2133^{\prime} 1^{\prime} 2^{\prime}}$ represents a laser beam passing along the arms from left to right; while, $\overleftarrow{3122^{\prime} 1^{\prime} 3^{\prime}}$ from right to left. $\alpha_{1}: \overrightarrow{{ }_{1} 2_{3} 1_{2} 3_{1}} \overleftrightarrow{{ }_{1}^{\prime}{ }_{3} 1_{2}^{\prime} 3_{1}^{\prime}}$ represents the paths of two laser beams for the first-generation TDI, then by reversing we obtain $\alpha_{2}:{ }_{1} 3_{2}^{\prime} 1_{3}^{\prime} 2_{1}^{\prime} \overleftrightarrow{{ }_{1} 1_{2} 1_{3} 2_{1}}$. The path $\alpha_{1}$ is spliced with the path $\alpha_{2}$ and result in the path $\alpha 12-1$. Here, '[ ]' indicates where we insert $\alpha_{2}$ into $\alpha_{1}$ [50.

From Eq. 18 , we can write the time difference of the combination $\alpha 12-1$ as:

$$
\begin{aligned}
\Delta t_{\alpha 12-1}= & L_{2 ; 133^{\prime} 1^{\prime} 2^{\prime}}+L_{1 ; 33^{\prime} 1^{\prime} 2^{\prime}}+L_{3 ; 3^{\prime} 1^{\prime} 2^{\prime}}+L_{3^{\prime} ; 1^{\prime} 2^{\prime}}+L_{1^{\prime} ; 2^{\prime}}+L_{2^{\prime}} \\
& -L_{3}-L_{1 ; 3}-L_{2 ; 13}-L_{2^{\prime} ; 213}-L_{1^{\prime} ; 2^{\prime} 213}-L_{3^{\prime} ; 1^{\prime} 2^{\prime} 213} .
\end{aligned}
$$

Similarly, the path $\alpha_{2}: \overline{{ }_{1} 3_{2 \uparrow}^{\prime} 1_{3}^{\prime} 2_{1}^{\prime}} \overleftarrow{{ }_{1}{ }_{2} 1_{3} 2_{1}}$ can be split at the position of ' $\uparrow$ '. This results in a new path $\overrightarrow{{ }_{2} 1_{3}^{\prime} 2_{1}^{\prime}} \overleftrightarrow{3_{2} 1_{3} 2_{1}} \overrightarrow{{ }_{1}} 3_{2}^{\prime}$, which makes S2 as the starting and ending satellite. Stitching it with $\alpha_{1}$, we can obtain a new combination:

$$
\begin{aligned}
& \overrightarrow{{ }_{1} 2_{3} 1_{2} 3_{1}} \overleftarrow{{ }_{1} 2_{3}^{\prime} 1_{2}^{\prime} 3_{1}^{\prime}}+\overrightarrow{{ }_{2} 1_{3}^{\prime} 2_{1}^{\prime}} \overleftarrow{3_{2} 1_{3} 2_{1}} \overrightarrow{{ }_{1} 3_{2}^{\prime}} \Longrightarrow \overrightarrow{21\left[1^{\prime} 2^{\prime}\right.} \overleftarrow{312} \overrightarrow{\left.3^{\prime}\right] 3} \overleftarrow{2^{\prime} 1^{\prime} 3^{\prime}} \quad(\alpha 12-2) \\
& =y_{23 ; 11^{\prime} 2^{\prime} \overline{31}}+y_{12 ; 1^{\prime} 2^{\prime} \overline{31}}+y_{13 ; 2^{\prime} \overline{31}}+y_{21 ; \overline{31}}-y_{31 ; \overline{1}}-y_{12 ; \overline{1}} \\
& -y_{23}+y_{32 ; \overline{3^{\prime}} 2}+y_{31 ; \overline{33^{\prime}} 2}-y_{21 ; \overline{33^{\prime}} 2}-y_{13 ; 2^{\prime}} \overline{33^{\prime} 2}-y_{32 ; 1^{\prime} 2^{\prime}} \overline{33^{\prime}} 2 .
\end{aligned}
$$


Here, following the advancement rule introduced in [50, we adopt the advancement indices $\bar{l}$. For example,

$$
y_{32 ; \overline{3^{\prime} 2}}=y_{32}\left(t+L_{3^{\prime}}\left(t-L_{2}(t)\right)-L_{2}(t)\right) .
$$

In addition, the path $\alpha_{2}$ can be split to make S3 as the starting and ending satellite, which gives:

$$
\overrightarrow{{ }_{1} 2_{3} 1_{2} 3_{1}} \overleftarrow{{ }_{1} 2_{3}^{\prime} 1_{2}^{\prime} 3_{1}^{\prime}}+\overrightarrow{{ }_{3} 2_{1}^{\prime}} \overleftrightarrow{3_{2} 1_{3} 2_{1}} \overrightarrow{{ }_{1} 3_{2}^{\prime} 1_{3}^{\prime}} \Longrightarrow \overrightarrow{2\left[2^{\prime}\right.} \overleftarrow{312} \overrightarrow{\left.3^{\prime} 1^{\prime}\right] 132^{\prime} 1^{\prime} 3^{\prime}} \quad(\alpha 12-3)
$$

Fig. 8 gives the smallest (right panel) and the largest (left panel) time differences for the second-generation $\alpha$-type combinations.

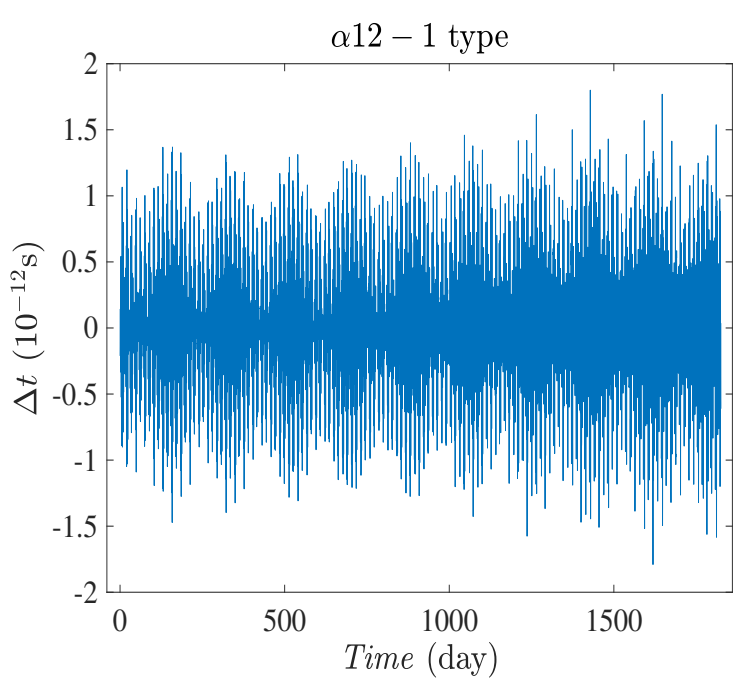

(a)

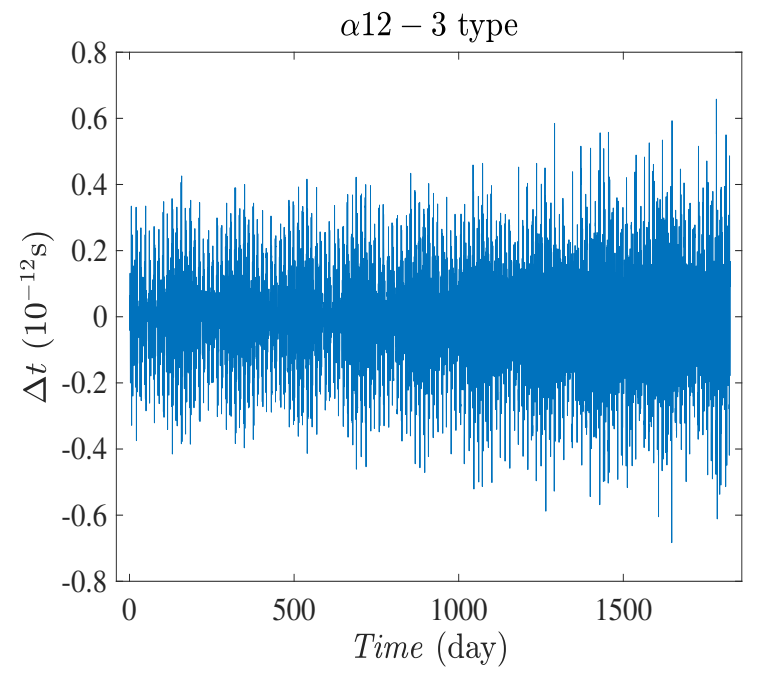

(b)

FIG. 8. The time differences for the second-generation $\alpha$-type combinations.

For the other second-generation TDI combinations, the results are:

$$
\begin{aligned}
& X: \overrightarrow{{ }_{1} 3_{2}^{\prime} 3_{1} 2_{3} 2_{1}^{\prime}} \overleftarrow{{ }_{1} 3_{2} 3_{1}^{\prime} 2_{3}^{\prime} 2_{1}}+\overrightarrow{{ }_{1} 2_{3} 2_{1}^{\prime} 3_{2}^{\prime} 3_{1}} \overleftarrow{{ }_{1}^{\prime} 2_{3}^{\prime} 2_{1} 3_{2} 3_{1}^{\prime}}
\end{aligned}
$$

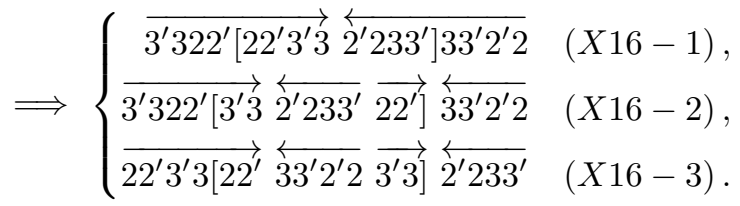

The path $X_{1}: \overrightarrow{{ }_{1} 3_{2}^{\prime} 3_{1} 2_{3} 2_{1}^{\prime}} \overleftarrow{3_{2} 3_{1}^{\prime} 2_{3}^{\prime} 2_{1}}$ is spliced with its reversed version $X_{2}: \overrightarrow{{ }_{1} 2_{3} 2_{1}^{\prime} 3_{2}^{\prime} 3_{1}} \overleftarrow{{ }_{1} 2_{3}^{\prime} 2_{1} 3_{2} 3_{1}^{\prime}}$, resulting in the path $X 16-1$. Also, the path $X_{1}: \overrightarrow{{ }_{1}^{\prime} 3_{2} 3_{1 \uparrow} 2_{3} 2_{1}^{\prime}} \overleftarrow{{ }_{1}{ }_{2} 3_{1}^{\prime} 2_{3}^{\prime} 2_{1}}$ can be split at the position of $\uparrow$, and generate the new path $X_{3}: \overrightarrow{22^{\prime}} \overleftarrow{33^{\prime} 2^{\prime} 2} \overrightarrow{3^{\prime} 3}$, which is spliced with the path $X_{2}$ to form the path $X 16-3$. The path $X_{2}$ can generate the new path $X_{4}: \overrightarrow{3^{\prime} 3} \overleftarrow{2^{\prime} 233^{\prime}} \overrightarrow{22^{\prime}}$, which is spliced with the path $X_{1}$ to form the path $X 16-2$. Among these three combinations, $X 16-1$ uses four directional arms which form a two-beam interferometers; While, $X 16-2$ and $X 16-3$ are four-beam interferometers.

Similar to Eq. 18 and Eq. 19, we can calculate the time differences for the second-generation $X$ combinations for TianQin. The smallest (right) and largest (left) time differences are shown in Fig. 9. As we will see below, the time differences of $X$ combinations are the largest comparing with in the $\alpha, U, P$ and $E$ combinations. This is because when inserting the frequency noise $C_{i}(t)$ into the combinations of ' $y$ ' for the $X$ (the expression similar to Eq. 18) and expanding in $L_{l}$ further than the canceled $L_{l}$ and $L_{l} \dot{L}_{m}$ terms, the next leading terms read $L^{2} \ddot{L}_{m}$ and the sums of these terms for the $X$ combinations are larger than the other combinations. 


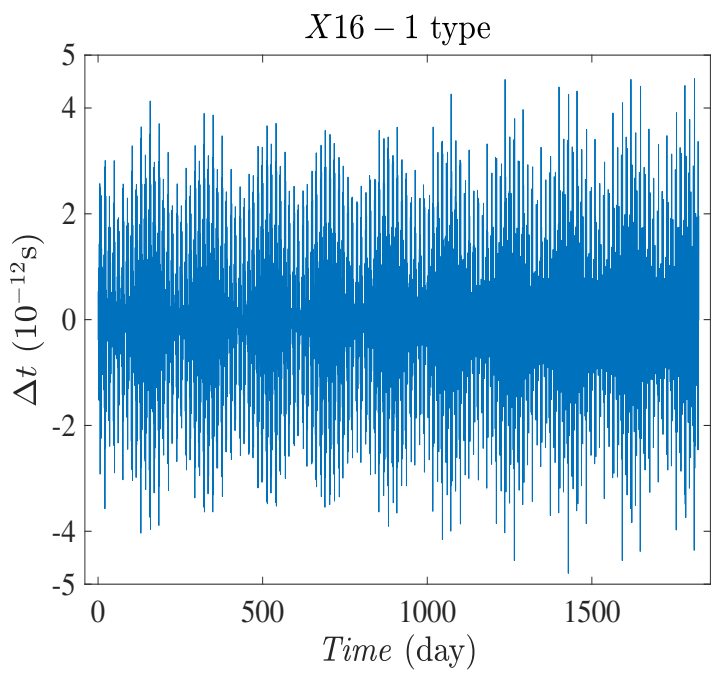

(a)

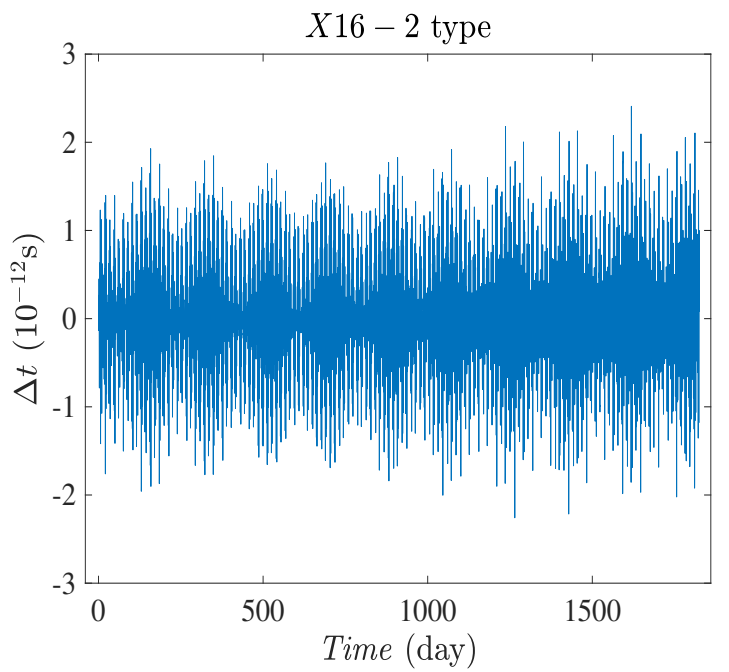

(b)

FIG. 9. The time differences for the second-generation $X$-type combinations.

$$
\begin{aligned}
& U: \overrightarrow{{ }_{3} 2_{1}^{\prime} 3_{2}^{\prime} 1_{3}^{\prime} 1_{2}} \overleftarrow{{ }_{2} 3_{1}^{\prime} 2_{3}^{\prime} 1_{2}^{\prime} 1_{3}}+\overrightarrow{{ }_{3} 1_{2} 1_{3}^{\prime} 2_{1}^{\prime} 3_{2}^{\prime}} \overleftarrow{{ }_{2} 1_{3} 1_{2}^{\prime} 3_{1}^{\prime} 2_{3}^{\prime}}
\end{aligned}
$$

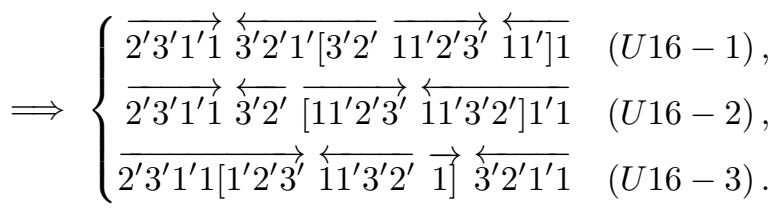

The path $U_{1}: \overrightarrow{{ }_{3}{ }_{1}^{\prime} 3_{2}^{\prime} 1_{3}^{\prime} 1_{2}} \overleftarrow{3_{2}^{\prime} 2_{3}^{\prime} 1_{2}^{\prime} 1_{3}}$ is spliced with its reversed version $U_{2}:{ }_{3} 1_{2} 1_{3}^{\prime} 2_{1}^{\prime} 3_{2}^{\prime} \quad \overleftarrow{{ }_{2} 1_{3} 1_{2}^{\prime} 3_{1}^{\prime} 2_{3}^{\prime}}$, resulting in the path $U 16-2$. Also, the path $U_{2}: \overrightarrow{1_{2} \uparrow 1_{3}^{\prime} 2_{1}^{\prime} 3_{2}^{\prime}} \overleftarrow{{ }_{2} 1_{3} 1_{2 \uparrow}^{\prime} 3_{1}^{\prime} 2_{3}^{\prime}}$ can be split at the position of $\uparrow$, and generate the new path $U_{3}: \quad \overrightarrow{1^{\prime} 2^{\prime} 3^{\prime}} \overleftarrow{11^{\prime} 3^{\prime} 2^{\prime}} \overrightarrow{1}$ and $U_{4}: \overleftarrow{3^{\prime} 2^{\prime}} \overleftrightarrow{11^{\prime} 2^{\prime} 3^{\prime}} \overleftarrow{11^{\prime}} . U_{3}$ is spliced with the path $U_{1}$ to form the path $U 16-3$. $U 16-1$ is obtained by $U_{1}$ and $U_{4}$. All the $U$-type combinations are four-beam interferometers. Here, four directional arms $2^{\prime}, 3^{\prime}, 1^{\prime}, 1$ are used. Fig. 10 gives the smallest (right panel) and the largest (left panel) time differences for the second-generation $U$-type combinations.

$$
\begin{aligned}
& P: \overrightarrow{{ }_{1} 3_{2}^{\prime} 1_{3}^{\prime} 1_{2}} \overleftarrow{{ }_{2} 3_{1}^{\prime}} \overrightarrow{{ }_{1} 2_{3}} \overleftrightarrow{{ }_{3} 1_{2}^{\prime} 1_{3} 2_{1}}+\overrightarrow{{ }_{1} 2_{3} 1_{2} 1_{3}^{\prime}} \overleftarrow{{ }_{3} 2_{1}} \overrightarrow{{ }_{1} 3_{2}^{\prime}} \overleftarrow{{ }_{2} 1_{3} 1_{2}^{\prime} 3_{1}^{\prime}}
\end{aligned}
$$

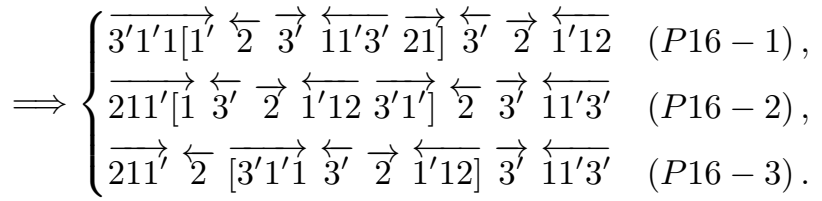

The path $P_{1}: \quad \overrightarrow{3_{2}^{\prime} 1_{3}^{\prime} 1_{2}} \overleftarrow{3_{3}^{\prime}} \overrightarrow{{ }_{1} 2_{3}} \overleftarrow{{ }_{3} 1_{2}^{\prime} 1_{3} 2_{1}}$ is spliced with its reversed version $P_{2}: \quad \overrightarrow{{ }_{1} 2_{3} 1_{2} 1_{3}^{\prime}} \overleftarrow{2_{2} 2_{1}} \overrightarrow{3_{2}^{\prime}} \overleftrightarrow{{ }_{2} 1_{3} 1_{2}^{\prime} 3_{1}^{\prime}}$, resulting in the path $P 16-3$. Also, the path $P_{2}: \overrightarrow{2_{3} 1_{2 \uparrow} 1_{3}^{\prime}} \overleftarrow{{ }_{3}{ }_{1}} \overrightarrow{{ }_{1} 3_{2}^{\prime}} \overleftarrow{{ }_{2}} \overleftarrow{1_{3} 1_{2}^{\prime} 3_{1}^{\prime}}$ can be split at the position of $\uparrow$, and generate the new path $P_{3}: \overrightarrow{1^{\prime}} \overleftarrow{2} \overrightarrow{3^{\prime}} \overleftarrow{11^{\prime} 3^{\prime}} \overrightarrow{21}$, which is spliced with the path $P_{1}$ to form $P 16-1$. From $P_{1}$ we can derive $P_{4}: \overrightarrow{1} \overleftarrow{3^{\prime}} \overrightarrow{2} \overleftarrow{1^{\prime} 12} \overrightarrow{3^{\prime} 1^{\prime}}$, which is spliced with the path $P_{2}$ to form $P 16-2$. Fig. 11 gives the smallest (right panel) and the largest (left panel) time differences for the second-generation $P$-type combinations. 


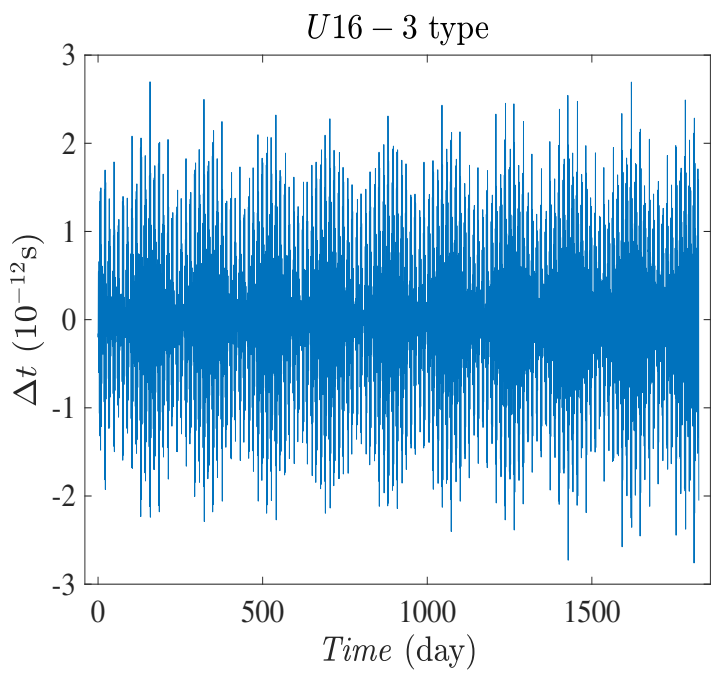

(a)

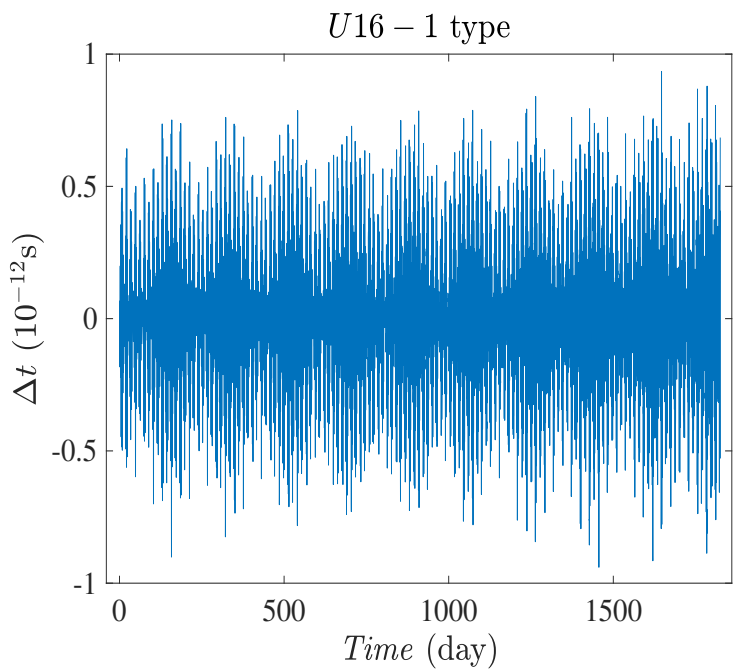

(b)

FIG. 10. The time differences for the second-generation $U$-type combinations.

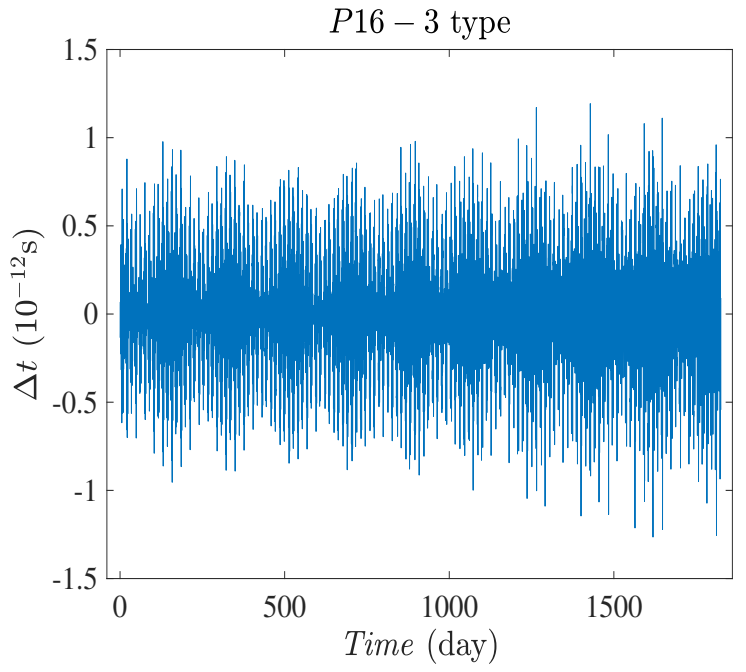

(a)

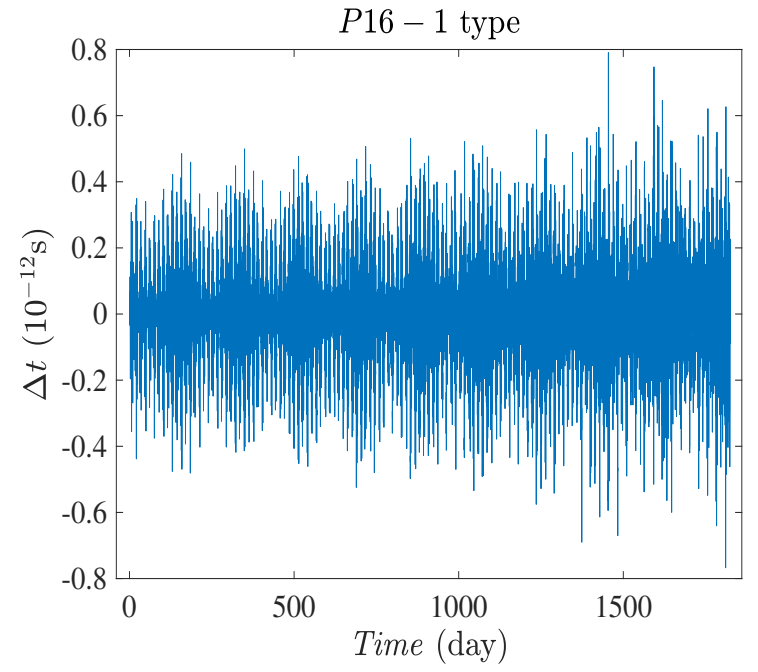

(b)

FIG. 11. The time differences for the second-generation $P$-type combinations.

$$
\begin{aligned}
& E: \overrightarrow{{ }_{2} 1_{3}^{\prime} 1_{2} 3_{1}} \overleftarrow{{ }_{1} 2_{3}^{\prime} 1_{2}^{\prime} 1_{3}} \overrightarrow{{ }_{3} 2_{1}^{\prime}} \overleftarrow{{ }_{1} 3_{2}}+\overrightarrow{{ }_{2} 3_{1}} \overleftarrow{{ }_{1} 2_{3}^{\prime}} \overrightarrow{1_{3} 1_{2}^{\prime} 2_{1}^{\prime}} \overleftarrow{{ }_{3} 3_{2} 1_{3} 1_{2}^{\prime}}
\end{aligned}
$$

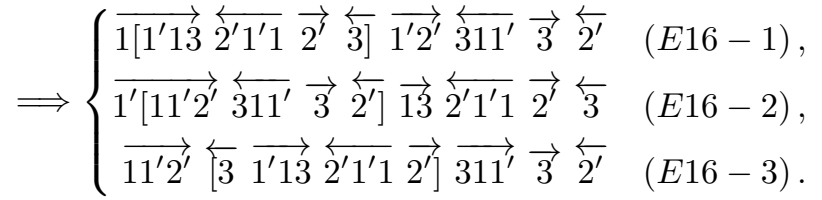

By reversing the interference arm of $E_{1}: \overrightarrow{{ }_{2} 1_{3}^{\prime} 1_{2} 3_{1}} \overleftarrow{2_{3}^{\prime} 1_{2}^{\prime} 1_{3}} \overrightarrow{2_{2} 2_{1}^{\prime}} \overleftarrow{3_{2}}$, we can obtain $E_{2}: \overrightarrow{{ }_{2} 3_{1}} \overleftarrow{2_{1}^{\prime}} \overrightarrow{1_{3} 1_{2} 1_{3}^{\prime} 2_{1}^{\prime}} \overleftarrow{3_{2} 3_{2} 1_{3} 1_{2}^{\prime}}$. $E_{2}: \overrightarrow{{ }_{2} 3_{1}} \overleftarrow{2_{2 \uparrow}^{\prime}} \overrightarrow{{ }_{3} 1_{2} 1_{3}^{\prime} 2_{1}^{\prime}} \overleftarrow{3_{2} 1_{3} 1_{2}^{\prime}}$ can be split at the position of $\uparrow$, and generate the new path $E_{3}: \overrightarrow{11^{\prime} 2^{\prime}} \overleftarrow{311^{\prime}} \overrightarrow{3} \overleftarrow{2^{\prime}}$, which is spliced with the path $E_{1}$ to form the path of $E 16-1$ and $E 16-2$. Also, the path $E_{1}$ can generate the new path of $E_{4}: \overleftarrow{3} \overrightarrow{1^{\prime} 13} \overleftarrow{2^{\prime} 1^{\prime} 1} \overleftarrow{2^{\prime}}$, which is spliced with the path of $E_{3}$ to form the path $E 16-3$. The combinations of $E$-type and $P$-type both form eight-beam interferometers. Fig. 12 gives the smallest (right panel) and the largest (left panel) 
time differences for the second-generation $E$-type combinations.

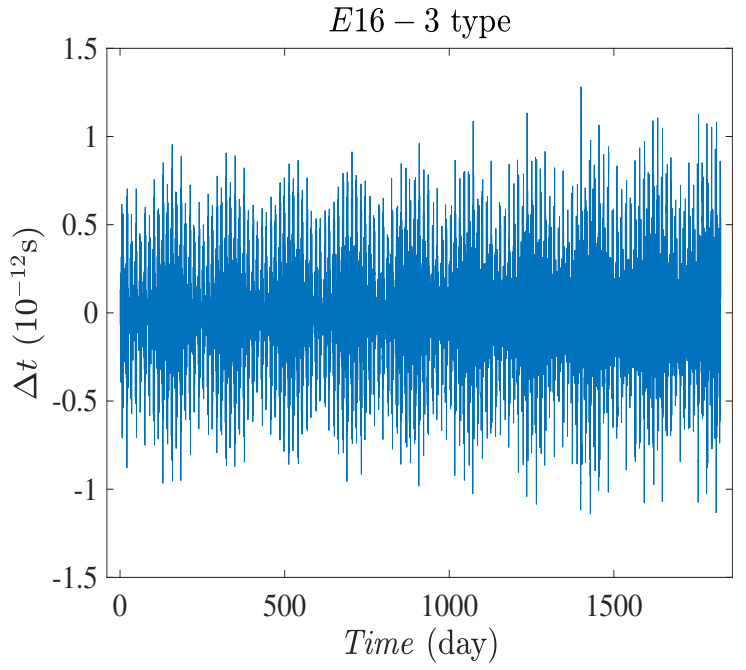

(a)

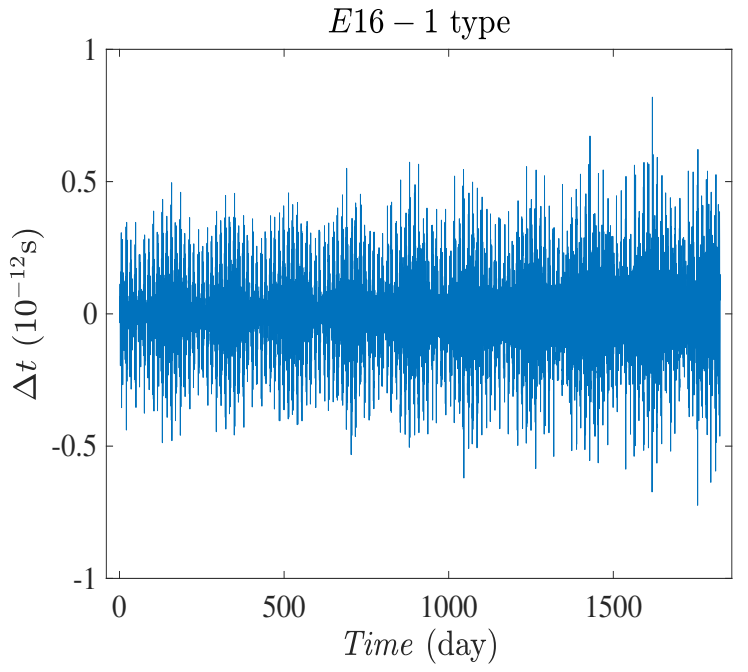

(b)

FIG. 12. The time differences for the second-generation E-type combinations.

As we can see from the results above, the eight-beam interferometers are better than the other second-generation TDI combinations. The time differences of all the second-generation TDI data combinations are below $5 \times 10^{-12} \mathrm{~s}$. Similar to Fig. 7. the results for the second-generation combinations, taking $\alpha 12-1$ and $X 16-1$ as examples, are shown in Fig. 13. Here, the secondary noise PSDs $S_{\alpha 12-1}(f)=4 \sin ^{2}(3 \pi f L) S_{\alpha}(f)$ and $S_{X 16-1}(f)=4 \sin ^{2}(4 \pi f L) S_{X}(f)$ [76] are adopted. We can see that the time differences of the second-generation data combinations $\left(\sim 10^{-12} \mathrm{~s}\right)$ are substantially lower than the requirements $\left(>10^{-10} \mathrm{~s}\right)$, thus they are guaranteed to suppress the laser frequency noise well below the secondary noises for TianQin in the concerned frequencies.

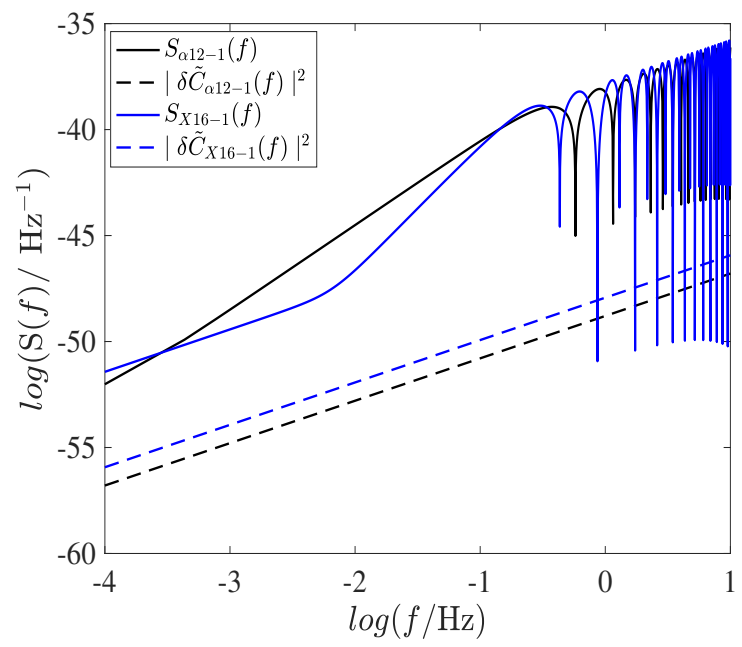

(a)

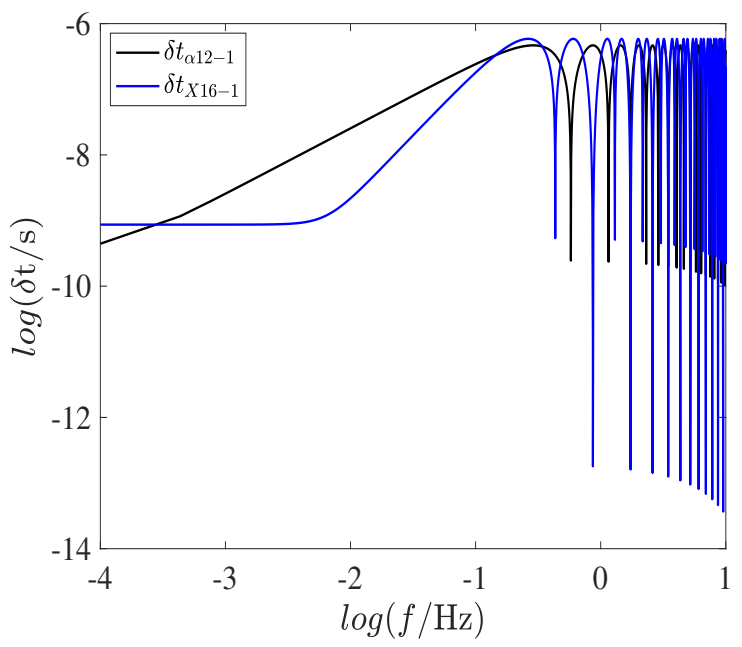

(b)

FIG. 13. Left panel shows the secondary noise (solid line) and the residual laser frequency noise (dotted line) for the second-generation $\alpha 12-1$ and X16-1 combinations. Right panel shows the required time differences for the corresponding combinations. 


\section{CONCLUSION AND DISCUSSIONS}

Using the numerically optimized orbit that shows realistic features of the satellite constellation, we investigated the time differences of the symmetric interference paths, as a measure of residual laser frequency noise, of various first- and second-generation TDI data combinations for TianQin. We found that while the second-generation TDI with a typical time differences of $10^{-12} \mathrm{~s}$ is guaranteed to be valid for laser frequency noise suppression, the first-generation TDI is possible to be competent for GW signals at frequencies $\lesssim 10^{-3} \mathrm{~Hz}$ and $\gtrsim 10^{-1} \mathrm{~Hz}$ (given the raw laser frequency noise of $10 \mathrm{~Hz} / \sqrt{\mathrm{Hz}}$ ), which cover the coalescence of the SMBHB with a redshifted total mass $>10^{6} M_{\odot} 24$ for the former or the inspiral of the stellar-mass black hole binary (e.g., GW150914) $\lesssim 1$ yr before its final merger [28] for the latter. The first-generation TDI combinations will become fully useful with improved stabilization of the raw laser frequency noise down to $\approx 1 \mathrm{~Hz} / \sqrt{\mathrm{Hz}}$ in $10^{-3}-10^{-2} \mathrm{~Hz}$. This further stabilization of laser frequency can be possibly achieved through suppressing the fluctuation of the Pound-Drever-Hall error signal offset, thermal stabilization at the zero-crossing temperature of the optical bench coefficient of thermal expansion, upgrading the automatic functions of the digital controller, etc. $77+79$. The first-generation TDI combinations, when implemented, will simplify the data analysis procedure and reduce the computational cost, since they employ half of the single-link data than the corresponding second-generation ones.

The current work serves as our first step towards building an end-to-end TDI simulation for TianQin. Here, we only discussed the inter-satellite measurements ' $y$ '. In reality, when each laser on the two optical benches housed in a satellite are unlocked or the acceleration noises of the optical benches are concerned, the intra-satellite measurements ' $z$ ' need to be included in the simulation, although which are not subjected to the orbital effects of the satellites discussed here. The simulated TDI data combinations, realistic noises from various subsystems, interplanetary and relativistic effects on the optical path, etc. will be taken as the input of subsequent GW data analysis for various astrophysical sources. These will be investigated in our future study.

\section{ACKNOWLEDGMENTS}

Y.W. is supported by the National Natural Science Foundation of China (NSFC) under Grants No. 11973024 and No. 11690021, and Guangdong Major Project of Basic and Applied Basic Research (Grant No. 2019B030302001). The contribution of S.C.H. to this paper is supported by NSFC under Grants No. 11873098. X.Z. is supported by NSFC Grant No. 11805287. W.S. acknowledges the support from the NSFC under Grant No. 11803008 and National Key Research and Development Program of China (Grant No. 2020YFC2201201). We thank the anonymous referee for helpful comments and suggestions.

[1] B. P. Abbott and et al. (LIGO Scientific Collaboration and Virgo Collaboration). Observation of Gravitational Waves from a Binary Black Hole Merger. Physical Review Letters, 116(6):061102, February 2016. doi:10.1103/PhysRevLett.116.061102

[2] B. P. Abbott and et al. (LIGO Scientific Collaboration and Virgo Collaboration). GWTC-1: A Gravitational-Wave Transient Catalog of Compact Binary Mergers Observed by LIGO and Virgo during the First and Second Observing Runs. Physical Review X, 9(3):031040, July 2019. doi:10.1103/PhysRevX.9.031040

[3] R. Abbott and et al. (LIGO Scientific Collaboration and Virgo Collaboration). GWTC-2: Compact Binary Coalescences Observed by LIGO and Virgo During the First Half of the Third Observing Run. arXiv e-prints, art. arXiv:2010.14527, October 2020.

[4] B. P. Abbott and et al. (LIGO Scientific Collaboration and Virgo Collaboration). GW170817: Observation of Gravitational Waves from a Binary Neutron Star Inspiral. Phys. Rev. Lett., 119:161101, Oct 2017. doi:10.1103/PhysRevLett.119.161101. URL https://link.aps.org/doi/10.1103/PhysRevLett.119.161101.

[5] Kentaro Somiya. Detector configuration of KAGRA-the japanese cryogenic gravitational-wave detector. Classical and Quantum Gravity, 29(12):124007, jun 2012. doi:10.1088/0264-9381/29/12/124007. URL https://doi.org/10.1088\% 2F0264-9381\%2F 29\%2F $12 \% 2 \mathrm{~F} 124007$.

[6] P. Amaro-Seoane, H. Audley, S. Babak, J. Baker, E. Barausse, and et al. Laser Interferometer Space Antenna. arXiv e-prints, February 2017.

[7] J. Luo, L.-S. Chen, H.-Z. Duan, Y.-G. Gong, S. Hu, J. Ji, Q. Liu, J. Mei, V. Milyukov, M. Sazhin, C.-G. Shao, V. T. Toth, H.-B. Tu, Y. Wang, Y. Wang, H.-C. Yeh, M.-S. Zhan, Y. Zhang, V. Zharov, and Z.-B. Zhou. TianQin: a spaceborne gravitational wave detector. Classical and Quantum Gravity, 33(3):035010, February 2016. doi:10.1088/02649381/33/3/035010

[8] S. Kawamura, M. Ando, N. Seto, S. Sato, T. Nakamura, and et al. The Japanese space gravitational wave antenna: DECIGO. Classical and Quantum Gravity, 28(9):094011, May 2011. doi:10.1088/0264-9381/28/9/094011 
[9] W. T. Ni. ASTROD Mission Concept and Measurement of the Temporal Variation in the Gravitational Constant. In Y. M. Cho, C. H. Lee, and S.-W. Kim, editors, Pacific Conference on Gravitation and Cosmology, page 309, 1998.

[10] M. Tinto, D. Debra, S. Buchman, and S. Tilley. glisa: geosynchronous laser interferometer space antenna concepts with off-the-shelf satellites. Review of Scientific Instruments, 86(1):330, 2015.

[11] X. Gong, Y.-K. Lau, S. Xu, P. Amaro-Seoane, S. Bai, X. Bian, Z. Cao, G. Chen, X. Chen, Y. Ding, P. Dong, W. Gao, G. Heinzel, M. Li, S. Li, F. Liu, Z. Luo, M. Shao, R. Spurzem, B. Sun, W. Tang, Y. Wang, P. Xu, P. Yu, Y. Yuan, X. Zhang, and Z. Zhou. Descope of the ALIA mission. In Journal of Physics Conference Series, volume 610 of Journal of Physics Conference Series, page 012011, May 2015. doi:10.1088/1742-6596/610/1/012011.

[12] C. Cutler and J. Harms. BBO and the Neutron-Star-Binary Subtraction Problem. Physics, 73(4):1405-1408, 2006.

[13] K. Danzmann (the LISA Study Team). ESA System and Technology Study Report No. ESA-SCI. 2000.

[14] M. Colpi, A.-C. Fabian, M. Guainazzi, P. McNamara, L. Piro, and N. Tanvir. Athena-lisa synergies. February 2019.

[15] M. Armano, H. Audley, G. Auger, J. T. Baird, M. Bassan, P. Binetruy, M. Born, D. Bortoluzzi, N. Brandt, M. Caleno, L. Carbone, A. Cavalleri, A. Cesarini, G. Ciani, G. Congedo, A. M. Cruise, K. Danzmann, and et al. Sub-femto- $g$ free fall for space-based gravitational wave observatories: Lisa pathfinder results. Phys. Rev. Lett., 116:231101, Jun 2016. doi:10.1103/PhysRevLett.116.231101. URL http://link.aps.org/doi/10.1103/PhysRevLett.116.231101.

[16] M. Armano, H. Audley, J. Baird, P. Binetruy, M. Born, and et al. Beyond the Required LISA Free-Fall Performance: New LISA Pathfinder Results down to $20 \mu \mathrm{Hz}$. Phys. Rev. Lett., 120(6):061101, Feb 2018. doi:10.1103/PhysRevLett.120.061101.

[17] Klaus Abich, Alexander Abramovici, Bengie Amparan, Andreas Baatzsch, Brian Bachman Okihiro, and et al. In-Orbit Performance of the GRACE Follow-on Laser Ranging Interferometer. Phys. Rev. Lett., 123(3):031101, July 2019. doi: 10.1103/PhysRevLett.123.031101

[18] T. E. Strohmayer. High-Resolution X-Ray Spectroscopy of RX J0806.3+1527 with Chandra. The Astrophysical Journal Letters, 679:L109, June 2008. doi:10.1086/589439.

[19] X.-C. Hu, X.-H. Li, Y. Wang, W.-F. Feng, M.-Y. Zhou, Y.-M. Hu, S.-C. Hu, J.-W. Mei, and C.-G. Shao. Fundamentals of the orbit and response for TianQin. Classical and Quantum Gravity, 35(9):095008, May 2018. doi:10.1088/1361-6382/aab52f.

[20] B.-B. Ye, X. Zhang, M.-Y. Zhou, Y. Wang, H.-M. Yuan, D. Gu, Y. Ding, J. Zhang, J. Mei, and J. Luo. Optimizing orbits for TianQin. International Journal of Modern Physics D, 28:1950121, July 2019. doi:10.1142/S0218271819501219

[21] Zhuangbin Tan, Bobing Ye, and Xuefeng Zhang. Impact of orbital orientations and radii on TianQin constellation stability. International Journal of Modern Physics D, 29(8):2050056, January 2020. doi:10.1142/S021827182050056X

[22] Bobing Ye, Xuefeng Zhang, Yanwei Ding, and Yunhe Meng. Eclipse Avoidance in TianQin Orbit Selection. arXiv e-prints, art. arXiv:2012.03269, December 2020.

[23] Xuefeng Zhang, Chengjian Luo, Lei Jiao, Bobing Ye, Huimin Yuan, Lin Cai, Defeng Gu, Jianwei Mei, and Jun Luo. Effect of Earth-Moon's gravity on TianQin's range acceleration noise. arXiv e-prints, art. arXiv:2012.03264, December 2020.

[24] W.-F. Feng, H.-T. Wang, X.-C. Hu, Y.-M. Hu, and Y. Wang. Preliminary study on parameter estimation accuracy of supermassive black hole binary inspirals for TianQin. Phys. Rev. D, 99(12):123002, June 2019. doi:10.1103/PhysRevD.99.123002

[25] H.-T. Wang, Z. Jiang, A. Sesana, E. Barausse, S.-J. Huang, Y.-F. Wang, W.-F. Feng, Y. Wang, Y.-M. Hu, J. Mei, and J. Luo. Science with the TianQin observatory: Preliminary results on massive black hole binaries. Phys. Rev. D, 100(4): 043003, August 2019. doi:10.1103/PhysRevD.100.043003

[26] C. Shi, J. Bao, H.-T. Wang, J.-d. Zhang, Y.-M. Hu, A. Sesana, E. Barausse, J. Mei, and J. Luo. Science with the TianQin observatory: Preliminary results on testing the no-hair theorem with ringdown signals. Phys. Rev. D, 100(4):044036, August 2019. doi:10.1103/PhysRevD.100.044036

[27] J. Bao, C. Shi, H. Wang, J.-d. Zhang, Y. Hu, J. Mei, and J. Luo. Constraining modified gravity with ringdown signals: An explicit example. Phys. Rev. D, 100(8):084024, October 2019. doi:10.1103/PhysRevD.100.084024

[28] Shuai Liu, Yi-Ming Hu, Jian-dong Zhang, and Jianwei Mei. Science with the TianQin observatory: Preliminary results on stellar-mass binary black holes. Phys. Rev. D, 101:103027, May 2020. doi:10.1103/PhysRevD.101.103027 URL https: //link.aps.org/doi/10.1103/PhysRevD.101.103027.

[29] Hui-Min Fan, Yi-Ming Hu, Enrico Barausse, Alberto Sesana, Jian-dong Zhang, Xuefeng Zhang, Tie-Guang Zi, and Jianwei Mei. Science with the TianQin observatory: Preliminary result on extreme-mass-ratio inspirals. Phys. Rev. D, 102:063016, Sep 2020. doi:10.1103/PhysRevD.102.063016. URL https://link.aps.org/doi/10.1103/PhysRevD.102.063016

[30] Shun-Jia Huang, Yi-Ming Hu, Valeriya Korol, Peng-Cheng Li, Zheng-Cheng Liang, Yang Lu, Hai-Tian Wang, Shenghua $\mathrm{Yu}$, and Jianwei Mei. Science with the TianQin Observatory: Preliminary results on Galactic double white dwarf binaries. Phys. Rev. D, 102:063021, Sep 2020. doi:10.1103/PhysRevD.102.063021. URL https://link.aps.org/doi/10.1103/ PhysRevD.102.063021.

[31] Jun Luo, Yan-Zheng Bai, Lin Cai, Bin Cao, Wei-Ming Chen, and et al. The first round result from the TianQin-1 satellite. Classical and Quantum Gravity, 37(18):185013, September 2020. doi:10.1088/1361-6382/aba66a

[32] Fangchao Yang, Yanzheng Bai, Wei Hong, Honggang Li, Li Liu, Timothy J. Sumner, Quanfeng Yang, Yujie Zhao, and Zebing Zhou. Investigation of charge management using UV LED device with a torsion pendulum for TianQin. Classical and Quantum Gravity, 37(11):115005, June 2020. doi:10.1088/1361-6382/ab8489

[33] Wei Su, Yan Wang, Ze-Bing Zhou, Yan-Zheng Bai, Yang Guo, Chen Zhou, Tom Lee, Ming Wang, Ming-Yue Zhou, Tong Shi, Hang Yin, and Bu-Tian Zhang. Analyses of residual accelerations for TianQin based on the global MHD simulation. Classical and Quantum Gravity, 37(18):185017, September 2020. doi:10.1088/1361-6382/aba181.

[34] Lingfeng Lu, Ying Liu, Huizong Duan, Yuanze Jiang, and Hsien-Chi Yeh. Numerical simulations of the wavefront distortion of inter-spacecraft laser beams caused by solar wind and magnetospheric plasmas. Plasma Science and Technology, 22(11): 115301, November 2020. doi:10.1088/2058-6272/abab69

[35] Jianwei Mei, Yan-Zheng Bai, Jiahui Bao, Enrico Barausse, Lin Cai, and et al. The TianQin project: Current 
progress on science and technology. Progress of Theoretical and Experimental Physics, 08 2020. ISSN 2050-3911. doi: 10.1093/ptep/ptaa114 URL https://doi.org/10.1093/ptep/ptaa114. ptaa114.

[36] B. S. Sathyaprakash and B. F. Schutz. Physics, Astrophysics and Cosmology with Gravitational Waves. Living Reviews in Relativity, 12:2, March 2009. doi:10.12942/lrr-2009-2

[37] Yi-Ming Hu, Jianwei Mei, and Jun Luo. Science prospects for space-borne gravitational-wave missions. National Science Review, 4(5):683-684, 2017. doi:10.1093/nsr/nwx115. URL http://dx.doi.org/10.1093/nsr/nwx115.

[38] J. W. Armstrong, F. B. Estabrook, and H. D. Wahlquist. A search for sinusoidal gravitational radiation in the period range 30-2000 seconds. Astrophys. J., 318:536-541, July 1987. doi:10.1086/165390.

[39] J. W. Armstrong, F. B. Estabrook, and M. Tinto. Time-Delay Interferometry for Space-based Gravitational Wave Searches. Astrophys. J., 527:814-826, December 1999. doi:10.1086/308110.

[40] F. B. Estabrook, M. Tinto, and J. W. Armstrong. Time-delay analysis of LISA gravitational wave data: Elimination of spacecraft motion effects. Phys. Rev. D, 62(4):042002, August 2000. doi:10.1103/PhysRevD.62.042002

[41] M. Tinto and J. W. Armstrong. Cancellation of laser noise in an unequal-arm interferometer detector of gravitational radiation. Phys. Rev. D, 59(10):102003, May 1999. doi:10.1103/PhysRevD.59.102003.

[42] M. Tinto, J. W. Armstrong, and F. B. Estabrook. Discriminating a gravitational wave background from instrumental noise in the LISA detector. Phys. Rev. D, 63(2):021101(R), December 2000. doi:10.1103/PhysRevD.63.021101.

[43] M. Tinto, F. B. Estabrook, and J. W. Armstrong. Time-delay interferometry for LISA. Phys. Rev. D, 65(8):082003, April 2002. doi:10.1103/PhysRevD.65.082003

[44] D. A. Shaddock, M. Tinto, F. B. Estabrook, and J. W. Armstrong. Data combinations accounting for LISA spacecraft motion. Phys. Rev. D, 68(6):061303(R), September 2003. doi:10.1103/PhysRevD.68.061303

[45] M. Tinto, F. B. Estabrook, and J. W. Armstrong. Time delay interferometry with moving spacecraft arrays. Phys. Rev. D, 69(8):082001, April 2004. doi:10.1103/PhysRevD.69.082001

[46] K. R. Nayak and J.-Y. Vinet. Algebraic approach to time-delay data analysis for orbiting LISA. Phys. Rev. D, 70(10): 102003, November 2004. doi:10.1103/PhysRevD.70.102003

[47] M. Tinto and S. V. Dhurandhar. Time-Delay Interferometry. Living Reviews in Relativity, 8:4, July 2005. doi:10.12942/lrr2005-4

[48] T. A. Prince, M. Tinto, S. L. Larson, and J. W. Armstrong. LISA optimal sensitivity. Phys. Rev. D, 66(12):122002, December 2002. doi:10.1103/PhysRevD.66.122002

[49] N. J. Cornish and R. W. Hellings. The effects of orbital motion on LISA time delay interferometry. Classical and Quantum Gravity, 20:4851-4860, November 2003. doi:10.1088/0264-9381/20/22/009.

[50] M. Vallisneri. Geometric time delay interferometry. Phys. Rev. D, 72(4):042003, August 2005. 10.1103/PhysRevD.72.042003.

[51] S V Dhurandhar, K Rajesh Nayak, S Koshti, and J-Y Vinet. Fundamentals of the LISA stable flight formation. Classical and Quantum Gravity, 22(3):481-487, jan 2005. doi:10.1088/0264-9381/22/3/002 URL https://doi.org/10.1088\% 2F0264-9381\%2F $22 \% 2 \mathrm{~F} 3 \% 2 \mathrm{~F} 002$.

[52] M. Vallisneri. Synthetic LISA: Simulating time delay interferometry in a model LISA. Phys. Rev. D, 71(2):022001, January 2005. doi:10.1103/PhysRevD.71.022001

[53] C. Tang and W. Ni. CGC 2 Ephemeris Framework. Publications of Yunnan Observatory, 3:21, 2002.

[54] G. Wang and W.-T. Ni. Numerical simulation of time delay interferometry for eLISA/NGO. Classical and Quantum Gravity, 30(6):065011, March 2013. doi:10.1088/0264-9381/30/6/065011.

[55] S. V. Dhurandhar, W.-T. Ni, and G. Wang. Numerical simulation of time delay interferometry for a LISA-like mission with the simplification of having only one interferometer. Advances in Space Research, 51:198-206, January 2013. doi: 10.1016/j.asr.2012.09.017

[56] G. Wang and W.-t. Ni. Time-delay Interferometry for ASTROD-GW. Chinese Astronomy and Astrophysics, 36:211-228, April 2012. doi:10.1016/j.chinastron.2012.04.009

[57] G. Wang and W.-T. Ni. Orbit optimization and time delay interferometry for inclined ASTROD-GW formation with half-year precession-period. Chinese Physics B, 24(5):059501, May 2015. doi:10.1088/1674-1056/24/5/059501.

[58] Gang Wang, Wei-Tou Ni, and Wen-Biao Han. Sensitivity investigation for unequal-arm LISA and TAIJI: the first-generation time-delay interferometry optimal channels. arXiv e-prints, art. arXiv:2008.05812, August 2020.

[59] M. J. Sidi. Spacecraft dynamics and control: a practical engineering approach. Cambridge University, 1997.

[60] A. E. Roy. Orbital motion. Institute of Physics, 2005.

[61] V. A. Chobotov. Orbital Mechanics. American Institute of Aeronautics and Astronautics, 2002.

[62] W. M. Folkner, J. G. Williams, and D. H. Boggs. The Planetary and Lunar Ephemeris DE 421. Interplanetary Network Progress Report, 178:1-34, August 2009.

[63] C. M. Will. Theory and Experiment in Gravitational Physics. UK: Cambridge University Press, March 1993.

[64] K. Nordtvedt. Equivalence Principle for Massive Bodies. II. Theory. Physical Review, May 1968.

[65] C. M. Will and K. Nordtvedt, Jr. Conservation Laws and Preferred Frames in Relativistic Gravity. I. Preferred-Frame Theories and an Extended PPN Formalism. Astrophys. J., 177:757, November 1972. doi:10.1086/151754

[66] S. C. Hu, Y. H. Zhao, and J. H. Ji. Internal report of purple mountain observatory (unpublished). 2015.

[67] Russell Eberhart and James Kennedy. A new optimizer using particle swarm theory. In Micro Machine and Human Science, 1995. MHS'95., Proceedings of the Sixth International Symposium on, pages 39-43. IEEE, 1995.

[68] Soumya Mohanty. Swarm Intelligence Methods for Statistical Regression. Chapman and Hall/CRC, 2018.

[69] T. S. Weerathunga and S. D. Mohanty. Performance of particle swarm optimization on the fully-coherent all-sky search for gravitational waves from compact binary coalescences. Phys. Rev. D, 95(12):124030, June 2017. doi: 
10.1103/PhysRevD.95.124030

[70] M. E. Normandin, S. D. Mohanty, and T. S. Weerathunga. Particle swarm optimization based search for gravitational waves from compact binary coalescences: Performance improvements. Phys. Rev. D, 98(4):044029, August 2018. doi: 10.1103/PhysRevD.98.044029

[71] Y. Wang, S. D. Mohanty, and F. A. Jenet. A Coherent Method for the Detection and Parameter Estimation of Continuous Gravitational Wave Signals Using a Pulsar Timing Array. Astrophys. J., 795:96, November 2014. doi:10.1088/0004$637 \mathrm{X} / 795 / 1 / 96$

[72] Y. Wang, S. D. Mohanty, and F. A. Jenet. Coherent Network Analysis for Continuous Gravitational Wave Signals in a Pulsar Timing Array: Pulsar Phases as Extrinsic Parameters. Astrophys. J., 815:125, December 2015. doi:10.1088/0004$637 \mathrm{X} / 815 / 2 / 125$.

[73] X. X. Newhall. Numerical Representation of Planetary Ephemerides. Celestial Mechanics, 45:305, 1989.

[74] W. H. Press, S. A. Teukolsky, W. T. Vetterling, and B. P. Flannery. Numerical recipes. The art of scientific computing. Cambridge University, 2007.

[75] Daniel A. Shaddock. Operating LISA as a Sagnac interferometer. Phys. Rev. D, 69(2):022001, January 2004. doi: 10.1103/PhysRevD.69.022001.

[76] Andrzej Królak, Massimo Tinto, and Michele Vallisneri. Optimal filtering of the LISA data. Phys. Rev. D, 70(2):022003, July 2004. doi:10.1103/PhysRevD.70.022003

[77] Y. Luo, H. Li, Y. Liang, H. Duan, Jingyi Zhang, and H. Yeh. A preliminary prototype of laser frequency stabilization for spaceborne interferometry missions. In 2016 European Frequency and Time Forum (EFTF), pages 1-4, 2016. doi: 10.1109/EFTF.2016.7477777

[78] K. Nicklaus, M. Herding, X. Wang, N. Beller, O. Fitzau, M. Giesberts, M. Herper, G. P. Barwood, R. A. Williams, P. Gill, H. Koegel, S. A. Webster, and M. Gohlke. High stability laser for next generation gravity missions. In Zoran Sodnik, Bruno Cugny, and Nikos Karafolas, editors, International Conference on Space Optics â ICSO 2014, volume 10563, pages 808 - 815. International Society for Optics and Photonics, SPIE, 2017. doi:10.1117/12.2304161. URL https://doi.org/10.1117/12.2304161

[79] Min Ming, Yingxin Luo, Yu-Rong Liang, Jing-Yi Zhang, Hui-Zong Duan, Hao Yan, Yuan-Ze Jiang, Ling-Feng Lu, Qin Xiao, Zebing Zhou, and Hsien-Chi Yeh. Ultraprecision intersatellite laser interferometry. International Journal of Extreme Manufacturing, 2(2):022003, may 2020. doi:10.1088/2631-7990/ab8864. URL https://doi.org/10.1088/2631-7990/ ab8864. 